\title{
Modeling for COVID-19 with the contacting distance
}

\author{
Zhihui Ma - Shufan Wang • Xuanru Lin • \\ Xiaohua Li • Xiaotao Han • Haoyang Wang • \\ Hua Liu
}

Received: 28 June 2021 / Accepted: 25 November 2021 / Published online: 16 January 2022

(C) The Author(s), under exclusive licence to Springer Nature B.V. 2021

\begin{abstract}
COVID-19 is a public health emergency for human beings and brings some very harmful consequences in social and economic fields. In order to model COVID-19 and develop the effective control measures, this paper proposes an SEIR-type epidemic model with the contacting distance between the healthy individuals and the asymptomatic or symptomatic infected individuals, and the immigration rate of the healthy individuals since the contacting distance and the immigration rate are two critical factors which determine the transmission of COVID-19. Firstly, the threshold values of the contacting distance and the immigration rate are obtained to analyze the presented model. Secondly, based on the data from January 10, 2020, to March 18, 2020, for Wuhan city, all parameters are estimated. Finally, based on the estimated parameters, the sensitivity analysis and the numerical study are conducted. The results show that the contacting distance and the immigration rate play an important role in controlling COVID-19. Meanwhile, the extinct lag decreases as the
\end{abstract}

Z. Ma $(\varangle) \cdot$ X. Lin $\cdot$ X. Li .

School of Mathematics and Statistics, Lanzhou University, Lanzhou 730000, Gansu, People's Republic of China

e-mail: mazhh@1zu.edu.cn

S. Wang $\cdot$ X. Han $\cdot$ H. Liu

School of Mathematics and Computer Science, Northwest

Minzu University, Lanzhou 730000, Gansu,

People's Republic of China

H. Wang

Faculty of Science, McMaster University, Hamilton, Ontario L8S4L8, Canada contacting distance increases and/or the immigration rate decreases. Our study could give some reasonable suggestions for the health officials and the public and provide a theoretical issue for globally controlling the COVID-19 pandemic.

Keywords COVID-19 • Contacting distance · Immigration rate $\cdot$ Sensitivity analysis $\cdot$ Numerical test Control measure

\section{Background}

Coronaviruses are the enveloped, single-stranded, positive-sense RNA viruses and belong to the family of Coronaviridae $[1,2]$. They could induce wildly respiratory infections, even though are accompanied by the relatively low mortality. Since their discovery and first characterization by the UK in 1965 [3], two largescale outbreaks have occurred and induced the public health events. For examples, the Severe Acute Respiratory Syndrome (SARS) in 2003 in mainland China [4], and the Middle East Respiratory Syndrome (MERS) in 2012 in Saudi Arabia [5-7]. These outbreaks have resulted in more than 8000 and 2200 confirmed SARS and MERS cases, respectively [1,8].

At the end of 2019, a third bigger outbreak occurred in the whole world. On December 29th 2019, four cases were reported by the Wuhan Municipal Health Commission (WMHC) and all confirmed cases are seemingly linked to the Huanan (Southern China) Seafood 
Wholesale Market (HSWM) [9-12]. These reported cases were firstly identified as the novel coronavirusinfected pneumonia, and then the World Health Organization (WHO) named this infectious disease as the coronavirus disease 2019, being simplified as COVID19, on February 11th, 2020 [10,13]. On January 20th, 2020, the Chinese government revised the law provisions about COVID-19 and defined it as class B agent. Public health officials announced a further revision to classify it as class A agent. Some non-pharmaceutical interventions, including the intensive contact tracing followed by quarantine of individuals potentially exposed to the infected peoples, the isolation of the symptomatic infected individuals and so all, were subsequently adopted, and then gave some positive effect on control of COVID-19.

COVID-19 has already caused a huge economic loss due to national lockdowns, travel restrictions, and global distractions of trade and manufacturing chains, and a large number of deaths due to the shortage of medical resources. Therefore, COVID-19 is a seriously public health emergency for the whole world and brings some very harmful consequences in social and economic fields [8-12,14]. In order to prevent and control COVID-19 effectively, lots of researchers have conducted to study COVID-19 based on medicine, epidemiology, biology and mathematics [2,9-12]. Li et al. [10] collected the confirmed information on demographic characteristics, exposure history and illness timelines of laboratory-confirmed cases that had been reported on January 22th, 2020. They described characteristics of the cases and estimated the key epidemiologic time-delay distributions (the $95 \mathrm{th}$ percentile of the distribution at 12.5 days), the epidemic doubling time (DT $=7.4$ days) and the basic reproductive number $\left(R_{0}=2.2(95 \% \mathrm{CI}, 1.4-3.9)\right)$. Fortunately, in the early period, this research has successfully characterized and predicted some critical properties of COVID19. However, as the development of COVID-19 and the adoptions of some effective prevention and control measures, COVID-19 should be re-estimated. Tang et al. [2] presented a general SEIR-type epidemiological model and estimated the basic reproduction number by means of mathematical modeling. Their results showed that the control reproduction number might be as high as 6.47 (95\% CI, 5.71-7.23). As recognized by the World Health Organization (WHO), the mathematical models, which are employed to describe COVID-19, play an important role for the disease prevention and control, and the policy formation.

The published researches have shown that the main transmission route of the COVID-19, including all epidemic diseases induced by the coronaviruses, is the respiratory transmission [2-4,15,16]. Hence, keeping the essential contacting distance between the healthy groups and the infected groups is very important for controlling COVID-19. Contacting distance refers to the extent to which people experience a sense of familiarity (nearness and intimacy) or unfamiliarity (fairness and difference) between themselves and people belonging to different social, ethnic, occupational and religious groups from their own [17-21]. The contacting distance describes the distance between different groups, and this notion includes all differences such as social class, race/ethnicity or sexuality, but also the fact that the different groups do not mix $[17,18]$. Therefore, in the mathematically modeling processes, the contacting distance between the healthy groups and the symptomatic infected ones is a critical parameter which determines the control measures for COVID19. Motivated by these, this paper will present an SEIR-type model incorporating the contacting distance between the healthy groups and the infected groups and mainly focus on exploiting the control measures for the COVID-19 based on the contacting distance. This paper will determine the threshold contacting distance between the healthy groups and the infected groups, and COVID-19 will be ultimately controlled when the contacting distance between the healthy groups and the infected groups is larger than this threshold contacting distance. Otherwise, COVID-19 may not be extinct. Here, the threshold contacting distance is a critical value, and COVID-19 will be extinct under this threshold contacting distance and COVID-19 may be endemic above this threshold contacting distance.

\section{Method}

In this section, an epidemic SEIR-type model with the contacting distance between the healthy groups and the symptomatic infected groups are presented to describe the spread of COVID-19 based on the following two steps.

Step 1. Proposing an SEIR-type epidemic model for COVID-19 with the incidence rate which will be presented in the later step 2 . 
$\left(A_{11}\right)$ Based on the spreading mechanism of COVID19 , the total populations are divided into five subclasses: the susceptible subpopulation $(S(t))$, the incubation subpopulation $(E(t))$, the asymptomatic infected subpopulation $\left(I_{A}(t)\right)$, the symptomatic infected subpopulation $(I(t))$ and the recovered subpopulation $(R(t))$.

$\left(A_{12}\right)$ The mean migrating rate of susceptible population for a local region, which describes the local outdoor movements of the susceptible individuals during the spread of COVID-19, is a constant recruitment $A$, which is defined in the interval $[0,+\infty)$. If the mean migrating rate is larger than zero and smaller than one, it means that individuals can only immigrate into the objective streets from their own home in their own local region. If the mean migrating rate is larger than one, it implies that some parts of individuals immigrate to the objective streets of the local region from the other regions.

$\left(A_{13}\right)$ The natural death rates of all subpopulation are proportional to their existing densities, and the proportional coefficient is $\mu$. The death rates of the asymptomatic infected subpopulation and symptomatic infected subpopulation are proportional to their existing densities, and the proportional coefficients are $\mu_{21}$ and $\mu_{22}$, respectively. The death rates of the incubation subpopulation could be omitted according to the report of the National Health Commission of the People's Republic of China (NHCPRC) and the World Health Organization (WHO).

$\left(A_{14}\right)$ The conversion rates from the incubation subpopulation to the asymptomatic infected subpopulation and the symptomatic infected subpopulation are proportional to the former's density and the proportional coefficients are $\gamma_{11}$ and $\gamma_{12}$, respectively. These conversion rates are mainly determined by the incubation period of COVID-19.

$\left(A_{15}\right)$ The recovery rate of the asymptomatic infected subpopulation and symptomatic infected subpopulation is proportional to their densities, and the proportional coefficients are $\gamma_{21}$ and $\gamma_{22}$, respectively. These are mainly determined by their autoimmunity and the medical level of the local hospitals. At present, the recovery rate of the symptomatic infected individuals prominently increases under the effective medical measures being adopted by the local government.

( $\left.A_{16}\right)$ The conversion rates from the susceptible subpopulation to the asymptomatic infected subpopulation and the asymptomatic infected subpopulation are defined as the incidence rate which are proposed in the following step 2 .

Step 2. According to our published work [19] which was done by some authors of this paper, we present the two incidence functions with the contacting distance explicitly for the asymptomatic and symptomatic infected populations induced by COVID-19 as the following form, respectively.

$\left(\operatorname{IF}\left(I_{A}\right)\right)$ The incidence functions with the contacting distance explicitly for the asymptomatic infected populations

$$
\operatorname{IF}\left(I_{A}\right)=\frac{\beta_{1}(1-d) S I_{A}}{1+\beta_{1}(1-d) h_{1} I_{A}},
$$

where $\beta_{1}$ is the natural infectious rate of the asymptomatic infected individuals who are carrying the coronavirus-2019, $h_{1}$ is the average effective contacting time of each asymptomatic infected individual carrying the coronavirus-2019.

Again, the term $\beta_{1}(1-d) I_{A}$ measures the positive infection force of COVID-19 for each susceptible individual, and the term $\frac{1}{1+\beta_{1}(1-d) h_{1} I_{A}}$ describes the inhibition effect when the number of the asymptomatic infected individuals increases. At present, this inhibition effect for COVID-19 is always existing since peoples are wearing the gauze masks and staying away from the crowds as far as possible under the media effect and fear effect for COVID-19. It is obtained that the term $\lim _{I_{A} \rightarrow+\infty} \frac{N\left(\dot{I}_{A}\right)}{T}=$ $\frac{1}{h_{1}}$ is constant, in other words, the infectious ability of COVID-19 will be saturated when the number of the asymptomatic infected individuals is large enough since the asymptomatic infected individuals are quarantined.

$(\operatorname{IF}(I))$ The incidence functions with the contacting distance explicitly for the symptomatic infected populations 


$$
\mathrm{IF}(I)=\frac{\beta_{2}(1-d) S I}{1+\beta(1-d) h_{2} I},
$$

where $\beta_{2}$ is the intrinsically infectious rate of the symptomatic individuals infected by COVID-19, $h_{2}$ is the average outdoor time of each symptomatic individuals infected by COVID-19.

It is reasonable to assume that $\beta_{1}<\beta_{2}$ and $h_{1}<h_{2}$.

Based on the above two steps, the epidemic model for COVID-19 incorporating the contacting distance is presented as the following form:

$$
\begin{aligned}
& \dot{S}(t)=A-\frac{\beta_{1}(1-d) S I_{A}}{1+\beta_{1}(1-d) h_{1} I_{A}} \\
& -\frac{\beta_{2}(1-d) S I}{1+\beta_{2}(1-d) h_{2} I}-\mu S \text {, } \\
& \dot{E}(t)=\frac{\beta_{1}(1-d) S I_{A}}{1+\beta_{1}(1-d) h_{1} I_{A}}+\frac{\beta_{2}(1-d) S I}{1+\beta_{2}(1-d) h_{2} I} \\
& -\mu E-\mu_{1} E-\gamma_{11} E-\gamma_{12} E, \\
& \dot{I}_{A}(t)=\gamma_{11} E-\mu I_{A}-\mu_{21} I_{A}-\gamma_{21} I_{A}, \\
& \dot{I}(t)=\gamma_{12} E-\mu I-\mu_{22} I-\gamma_{22} I, \\
& \dot{R}(t)=\mu_{1} E+\gamma_{21} I_{A}+\gamma_{22} I-\mu R,
\end{aligned}
$$

with the initial conditions:

$S(0) \geq 0, \quad E(0) \geq 0, \quad I_{A}(0) \geq 0$,

$I(0) \geq 0, \quad R(0) \geq 0$.

The epidemiological meanings of all parameters of model (2.3) are listed in the following Table 1.

Note that the recover variable $R(t)$ does not appear in the first four equations of model (2.3), this paper focuses on the following subsystem which mainly determines the dynamical behaviors of the original model system:

$$
\begin{aligned}
& \dot{S}(t)=A-\frac{\beta_{1}(1-d) S I_{A}}{1+\beta_{1}(1-d) h_{1} I_{A}} \\
& -\frac{\beta_{2}(1-d) S I}{1+\beta_{2}(1-d) h_{2} I}-\mu S \text {, } \\
& \dot{E}(t)=\frac{\beta_{1}(1-d) S I_{A}}{1+\beta_{1}(1-d) h_{1} I_{A}}+\frac{\beta_{2}(1-d) S I}{1+\beta_{2}(1-d) h_{2} I} \\
& -\mu E-\mu_{1} E-\gamma_{11} E-\gamma_{12} E, \\
& \dot{I}_{A}(t)=\gamma_{11} E-\mu I_{A}-\mu_{21} I_{A}-\gamma_{21} I_{A}, \\
& \dot{I}(t)=\gamma_{12} E-\mu I-\mu_{22} I-\gamma_{22} I \text {, }
\end{aligned}
$$

with the initial conditions:

$$
S(0) \geq 0, \quad E(0) \geq 0, \quad I_{A}(0) \geq 0, \quad I(0) \geq 0 .
$$

\section{Results}

\subsection{Basic property}

In order to guarantee the ecologically and epidemically realistic meanings of model (2.5) and model (2.6) with their own initial conditions, the positivity and boundness of all solutions of model (2.5) and model (2.6) with their own initial conditions should be validated.

Proposition 3.1.1 All solutions $\left(S(t), E(t), I_{A}(t)\right.$, $I(t), R(t))$ of model (2.5) with the initial conditions (2.6) are positive on the finite region for all $t>0$.

Proposition 3.1.2 All solutions $\left(S(t), E(t), I_{A}(t)\right.$, $I(t), R(t))$ of model (2.5) with the initial conditions (2.6) are bounded on the finite region in the following set $\Omega$, where

$$
\begin{aligned}
\Omega= & \left\{\left(S(t), E(t), I_{A}(t), I(t)\right), R(t)\right) \in R_{+}^{5} \mid \\
& \left.\quad 0<S(t)+E(t)+I_{A}(t)+I(t)\right)+R(t) \\
\leq & \left.\frac{A}{\mu}\right\} .
\end{aligned}
$$

Based on the above theorems, the presented model (2.5) with the initial conditions (2.6) is mathematically well-behaviored, and has its own epidemiological and ecological meanings. Hence, the realistic aspects and properties of model (2.5) with the initial conditions (2.6) are also guaranteed. Next, this paper focuses on the dynamical behaviors of model (2.5) with the initial conditions (2.6) and the theoretically controlling methods for the epidemic diseases.

\subsection{The basic reproduction number}

In order to describe the expected number of secondary cases produced by a typical infective individual while considering the intervention strategies, the reproduction number $R_{0}$ of model (2.5) is defined by the theory of the next-generation matrix [21]

$$
R_{0}=\rho\left(\left(\left.D F\right|_{P_{0}}\right) \bullet\left(\left.D V\right|_{P_{0}}\right)^{-1}\right),
$$


Table 1 The epidemiological meanings of parameters in model (2.3)

\begin{tabular}{ll}
\hline Parameter & Epidemiological meaning \\
\hline$\beta_{1}$ & The influx of the susceptible subpopulation. \\
$\beta_{2}$ & The intrinsically infectious rate of the asymptomatic infected individual. \\
$h_{1}$ & The intrinsically infectious rate of the symptomatic infected individual. \\
$h_{2}$ & The average outdoor time of each asymptomatic infected individual. \\
$\mu$ & The average outdoor time of each symptomatic infected individual. \\
$\mu_{1}$ & The natural death rates of all subpopulations. \\
$\mu_{21}$ & The recovery rate of the incubation subpopulation. \\
$\mu_{22}$ & The death rate of the asymptomatic infected individual induced by the coronavirus disease 2019 (COVID-19). \\
$\gamma_{11}$ & The death rate of the symptomatic infected individual induced by the coronavirus disease 2019 (COVID-19). \\
$\gamma_{12}$ & The transition rate from the incubation subpopulation to the asymptomatic infected subpopulation. \\
$\gamma_{21}$ & The transition rate from the incubation subpopulation to the symptomatic infected subpopulation. \\
$\gamma_{22}$ & The recovery rate of the asymptomatic infected subpopulation. \\
$d$ & The recovery rate of the symptomatic infected subpopulation. \\
\hline
\end{tabular}

in which $F$ is the regeneration matrix matrix and $V$ is the transition matrix of model (2.5).

In order to compute the reproduction number $R_{0}$ of model (2.5), let

$F=\left(\begin{array}{cc}\frac{\beta_{1}(1-d) S I_{A}}{1+\beta_{1}(1-d) h_{1} I_{A}}+\frac{\beta_{2}(1-d) S I}{1+\beta_{2}(1-d) h_{2} I} \\ 0 \\ 0 \\ 0 \\ 0\end{array}\right)$

and

$\left.\mathrm{DV}\right|_{P_{0}}=\left(\begin{array}{ccccc}\mu+\mu_{1}+\gamma_{11}+\gamma_{12} & 0 & 0 & 0 & 0 \\ -\gamma_{11} & \mu+\mu_{21}+\gamma_{21} & 0 & 0 & 0 \\ -\gamma_{12} & 0 & \mu+\mu_{22}+\gamma_{22} & 0 & 0 \\ -\mu_{1} & -\gamma_{21} & -\gamma_{22} & \mu & 0 \\ 0 & \frac{\beta_{1}(1-d) A}{\mu} & \frac{\beta_{2}(1-d) A}{\mu} & 0 & \mu\end{array}\right)$.

and

$$
V=\left(\begin{array}{c}
\frac{\beta_{1}(1-d) S I_{A}}{1+\beta_{1}(1-d) h_{1} I_{A}}+\frac{\beta_{2}(1-d) S I}{1+\beta_{2}(1-d) h_{2} I} \\
\left(\mu+\mu_{1}+\gamma_{11}+\gamma_{12}\right) E \\
\left(\mu+\mu_{21}+\gamma_{21}\right) I_{A}-\gamma_{11} E \\
\mu R-\mu_{1} E+\gamma_{21} I_{A}-\gamma_{22} I \\
\frac{\beta_{2}(1-d) S I_{A}}{1+\beta_{1}(1-d) h_{1} I_{A}}+\frac{\beta_{2}(1-d) S I}{1+\beta_{2}(1-d) h_{2} I}+\mu S-A
\end{array}\right) .
$$

Hence, it is obtained that

$\left.\mathrm{DF}\right|_{P_{0}}=\left(\begin{array}{ccccc}0 & \frac{\beta_{1}(1-d) A}{\mu} \frac{\beta_{2}(1-d) A}{\mu} & 0 & 0 \\ 0 & 0 & 0 & 0 & 0 \\ 0 & 0 & 0 & 0 & 0 \\ 0 & 0 & 0 & 0 & 0 \\ 0 & 0 & 0 & 0 & 0\end{array}\right)$


Thus, we have

$$
\left(\left.\mathrm{DV}\right|_{P_{0}}\right)^{-1}=\left(\begin{array}{ccccc}
\frac{1}{\mu+\mu_{1}+\gamma_{11}+\gamma_{12}} & 0 & 0 & 0 & 0 \\
\frac{1}{\left(\mu+\mu_{1}+\gamma_{11}+\gamma_{12}\right)\left(\mu+\mu_{21}+\gamma_{21}\right)} & \frac{1}{\mu+\mu_{21}+\gamma_{21}} & 0 & 0 & 0 \\
\frac{1}{\left(\mu+\mu_{1}+\gamma_{11}+\gamma_{12}\right)\left(\mu+\mu_{22}+\gamma_{22}\right)} & 0 & \frac{1}{\mu+\mu_{22}+\gamma_{22}} & 0 & 0 \\
a_{41} & \frac{\gamma_{21}}{\mu\left(\mu+\mu_{21}+\gamma_{21}\right)} \frac{\gamma_{22}}{\mu\left(\mu+\mu_{22}+\gamma_{22}\right)} & \frac{1}{\mu} & 0 \\
a_{51} & \frac{\beta_{1}(1-d) A}{\mu\left(\mu+\mu_{21}+\gamma_{21}\right)} \frac{\beta_{2}(1-d) A}{\mu\left(\mu+\mu_{22}+\gamma_{22}\right)} & 0 & \frac{1}{\mu}
\end{array}\right),
$$

in which

$$
\left\{\begin{array}{l}
a_{41}=\frac{\mu \mu_{1}\left(\mu+\mu_{21}+\gamma_{21}\right)\left(\mu+\mu_{22}+\gamma_{22}\right)+\mu \gamma_{11} \gamma_{21}\left(\mu+\mu_{22}+\gamma_{22}\right)+\mu \gamma_{12} \gamma_{22}\left(\mu+\mu_{21}+\gamma_{21}\right)}{|\mathrm{DV}|}>0, \\
a_{51}=-\frac{\beta_{1}(1-d) A \gamma_{11}\left(\mu+\mu_{22}+\gamma_{22}\right)+\beta_{2}(1-d) A \gamma_{12}\left(\mu+\mu_{21}+\gamma_{21}\right)}{|\mathrm{DV}|}<0 .
\end{array}\right.
$$

Therefore, the basic reproduction number is

$$
\begin{aligned}
R_{0}= & \rho\left(\left(\left.\mathrm{DF}\right|_{P_{0}}\right) \bullet\left(\left.\mathrm{DV}\right|_{P_{0}}\right)^{-1}\right) \\
= & \frac{(1-d) A}{\mu\left(\mu+\mu_{1}+\gamma_{11}+\gamma_{12}\right)} \\
& \left(\frac{\beta_{1} \gamma_{11}}{\mu+\mu_{21}+\gamma_{21}}+\frac{\beta_{2} \gamma_{12}}{\mu+\mu_{22}+\gamma_{22}}\right) .
\end{aligned}
$$

\subsection{The extinct threshold value}

In this section, the importantly interesting thing is to consider the dynamical behavior of the disease-free equilibrium since the main focus is to develop the controlling methods for COVID-19.

Clearly, the disease-free equilibrium point of model (2.5) $P_{0}$ is $\left(\frac{A}{\mu}, 0,0,0\right)$, and we mainly consider the dynamical behavior of the disease-free equilibrium point $E_{0}$.

The Jacobia matrix of model (2.5) at the disease-free equilibrium point $P_{0}$ is as follows

$$
J_{P_{0}}=\left(\begin{array}{cccc}
-\mu & 0 & -\frac{A_{4}(1-d)}{\gamma_{11}} & -\frac{A_{5}(1-d)}{\gamma_{12}} \\
0 & -A_{1} & \frac{A_{4}(1-d)}{\gamma_{11}} & \frac{A_{5}(1-d)}{\gamma_{12}} \\
0 & \gamma_{11} & -A_{2} & 0 \\
0 & \gamma_{12} & 0 & -A_{3}
\end{array}\right) .
$$

where

$$
\left\{\begin{array}{l}
A_{1}=\mu+\mu_{1}+\gamma_{11}+\gamma_{12}>0 \\
A_{2}=\mu+\mu_{21}+\gamma_{21}>0 \\
A_{3}=\mu+\mu_{22}+\gamma_{22}>0 \\
A_{4}=\frac{\beta_{1} \gamma_{11} A}{\mu}>0 \\
A_{5}=\frac{\beta_{2} \gamma_{12} A}{\mu}>0
\end{array}\right.
$$

Therefore, the characteristic equation of model (2.5) at the disease-free equilibrium point $P_{0}$ is presented by the following form:

$$
(\lambda+\mu)\left(\lambda^{3}+B_{1} \lambda^{2}+B_{2} \lambda+B_{3}\right)=0,
$$

in which

$$
\left\{\begin{aligned}
B_{1}= & A_{1}+A_{2}+A_{3}>0 \\
B_{2}= & A_{1} A_{2}+A_{1} A_{3}+A_{2} A_{3} \\
& \quad-A_{4}(1-d)-A_{5}(1-d) \\
B_{3}= & A_{1} A_{2} A_{3}-A_{3} A_{4}(1-d)-A_{2} A_{5}(1-d) .
\end{aligned}\right.
$$

According to Routh-Hurwitz rule, the disease-free equilibrium point is $P_{0}\left(\frac{A}{\mu}, 0,0,0\right)$ is locally asymptotically stable if and only if

$$
\left\{\begin{array}{l}
B_{1}>0, B_{3}>0, \\
B_{1} B_{2}-B_{3}>0
\end{array}\right.
$$


Next, two cases are considered:

$B_{3}>0$

$\Leftrightarrow A_{1} A_{2} A_{3}-\left(A_{3} A_{4}+A_{2} A_{5}\right)(1-d)>0$

$\Leftrightarrow \frac{1-d}{A_{1}}\left(\frac{A_{4}}{A_{2}}+\frac{A_{5}}{A_{3}}\right)<1$

$\Leftrightarrow \frac{(1-d) A}{\mu\left(\mu+\mu_{1}+\gamma_{11}+\gamma_{12}\right)}$

$\left(\frac{\beta_{1} \gamma_{11}}{\mu+\mu_{21}+\gamma_{21}}+\frac{\beta_{2} \gamma_{12}}{\mu+\mu_{22}+\gamma_{22}}\right)<1$

$\Leftrightarrow R_{0}<1$.

Again, we have

$$
\begin{aligned}
B_{1} & B_{2}-B_{3}>0 \\
\Leftrightarrow & \left(A_{1}+A_{2}+A_{3}\right)\left(A_{1} A_{3}+A_{2} A_{3}\right. \\
& \left.+A_{1} A_{2}-A_{4}(1-d)-A_{5}(1-d)\right) \\
& -\left(A_{1} A_{2} A_{3}-A_{3} A_{4}(1-d)-A_{2} A_{5}(1-d)\right)>0 \\
\Leftrightarrow & A_{1}^{2} A_{3}+2 A_{1} A_{2} A_{3}+A_{1}^{2} A \\
& +A_{2}^{2} A_{3}+A_{1} A_{2}^{2}+A_{1} A_{3}^{2}+A_{2} A_{3}^{2} \\
& -\left[\left(A_{1}+A_{2}\right) A_{4}+\left(A_{1}+A_{3}\right) A_{5}\right](1-d)>0 \\
\Leftrightarrow & \left(A_{1}+A_{3}\right)\left(A_{1} A_{3}+A_{2}\left(A_{1}+A_{2}+A_{3}\right)\right) \\
& -\left[\left(A_{1}+A_{2}\right) A_{4}+\left(A_{1}+A_{3}\right) A_{5}\right](1-d)>0 \\
\Leftrightarrow & 2 A_{1} A_{2} A_{3}+A_{1} A_{3}^{2}+A_{2}^{2} A_{3}+A_{2} A_{3}^{2}+A_{1} A_{2}^{2} \\
& -\left(A_{1} A_{4}(1-d)+A_{1} A_{5}(1-d)\right) \\
& -\left(A_{2} A_{4}(1-d)+A_{3} A_{5}(1-d)\right)>0 .
\end{aligned}
$$

If $R_{0}<1$, then we have

$$
\begin{aligned}
& 2 A_{1} A_{2} A_{3}+A_{1} A_{3}^{2}+A_{2}^{2} A_{3}+A_{2} A_{3}^{2}+A_{1} A_{2}^{2} \\
& \quad-\left(A_{1} A_{4}(1-d)+A_{1} A_{5}(1-d)\right) \\
& \quad-\left(A_{2} A_{4}(1-d)+A_{3} A_{5}(1-d)\right) \\
& > \\
& \quad\left(A_{1}+A_{2}\right)\left(A_{1}+A+3\right)>0 .
\end{aligned}
$$

Therefore, the disease-free equilibrium point is $P_{0}\left(\frac{A}{\mu}, 0,0,0\right)$ is locally asymptotically stable if and only if

$$
\begin{aligned}
& R_{0}<1 \Leftrightarrow d>1 \\
& -\frac{\mu\left(\mu+\mu_{1}+\gamma_{11}+\gamma_{12}\right)\left(\mu+\mu_{22}+\gamma_{22}\right)\left(\mu+\mu_{21}+\gamma_{21}\right)}{\beta_{1} \gamma_{11} A\left(\mu+\mu_{22}+\gamma_{22}\right)+\beta_{2} \gamma_{12} A\left(\mu+\mu_{21}+\gamma_{21}\right)}>0 .
\end{aligned}
$$

Based on the above analysis, we obtain the following results.

Proposition 3.3.1 Suppose that $\beta_{1} \gamma_{11} A\left(\mu+\mu_{22}+\right.$ $\left.\gamma_{22}\right)+\beta_{2} \gamma_{12} A\left(\mu+\mu_{21}+\gamma_{21}\right)>\mu\left(\mu+\mu_{1}+\gamma_{11}+\right.$ $\left.\gamma_{12}\right)\left(\mu+\mu_{22}+\gamma_{22}\right)\left(\mu+\mu_{21}+\gamma_{21}\right)$, we have

(1) If $R_{0}>1$ or

$d<1-\frac{\mu\left(\mu+\mu_{1}+\gamma_{11}+\gamma_{12}\right)\left(\mu+\mu_{22}+\gamma_{22}\right)\left(\mu+\mu_{21}+\gamma_{21}\right)}{\beta_{1 \gamma_{11}} A\left(\mu+\mu_{22}+\gamma_{22}\right)+\beta_{2} \gamma_{12} A\left(\mu+\mu_{21}+\gamma_{21}\right)}$, then the disease-free equilibrium point $P_{0}$ is unstable,
(2) If $R_{0}<1$ or

$d>1-\frac{\mu\left(\mu+\mu_{1}+\gamma_{11}+\gamma_{12}\right)\left(\mu+\mu_{22}+\gamma_{22}\right)\left(\mu+\mu_{21}+\gamma_{21}\right)}{\beta_{1} \gamma_{11} A\left(\mu+\mu_{22}+\gamma_{22}\right)+\beta_{2} \gamma_{12} A\left(\mu+\mu_{21}+\gamma_{21}\right)}$, then the disease-free equilibrium point $P_{0}$ is locally asymptotically stable.

Proposition 3.3.1 reveals that COVID-19 can be controlled while the contacting distance between the healthy individuals and the symptomatic infected individuals is larger than the threshold value $d^{*}=1-$ $\frac{\mu\left(\mu+\mu_{1}+\gamma_{11}+\gamma_{12}\right)\left(\mu+\mu_{22}+\gamma_{22}\right)\left(\mu+\mu_{21}+\gamma_{21}\right)}{\beta_{1} \gamma_{11} A\left(\mu+\mu_{22}+\gamma_{22}\right)+\beta_{2} \gamma_{12} A\left(\mu+\mu_{21}+\gamma_{21}\right)}$. This threshold value decreases with the decrease in the immigration rate of susceptible population.

\subsection{Model (2.5) without the asymptomatic infected population}

If the asymptomatic infected population is omitted since it is difficult to be found and the infection probability of the asymptomatic individuals is very small, then the model (2.5) will become the following forms

$$
\begin{aligned}
\dot{S}(t) & =A-\frac{\beta_{2}(1-d) S I}{1+\beta_{2}(1-d) h_{2} I}-\mu S, \\
\dot{E}(t) & =\frac{\beta_{2}(1-d) S I}{1+\beta_{2}(1-d) h_{2} I}-\mu E-\mu_{1} E-\gamma_{12} E, \\
\dot{I}(t) & =\gamma_{12} E-\mu I-\mu_{22} I-\gamma_{22} I,
\end{aligned}
$$

with the initial conditions:

$$
S(0) \geq 0, \quad E(0) \geq 0, \quad I(0) \geq 0 .
$$

According to Propositions 3.1.1 and 3.1.2, all solutions of model (3.11) with the initial conditions (3.12) are positive and bounded.

According to the computation of the basic reproduction number of model (2.5) $\tilde{R}_{0}$, the basic reproduction number of model (3.11) is

$$
\bar{R}_{0}=\frac{\beta_{2} \gamma_{12} A(1-d)}{\mu\left(\mu+\mu_{1}+\gamma_{12}\right)\left(\mu+\mu_{22}+\gamma_{22}\right)} .
$$

By simple computation, the disease-free equilibrium point of model (3.11) is $\tilde{P}_{0}\left(\frac{A}{\mu}, 0,0\right)$ and the corresponding endemic equilibrium point is $\tilde{P}(\tilde{S}, \tilde{E}, \tilde{I})$, where

$$
\left\{\begin{array}{l}
\tilde{S}=\frac{\beta_{2} \gamma_{12}(1-d)+\mu\left(\mu+\mu_{1}+\gamma_{12}\right)\left(\mu+\mu_{22}+\gamma_{22}\right)}{\beta_{2} \gamma_{12}(1-d)\left(\mu h_{2}+1\right)}, \\
\tilde{E}=\frac{\beta_{2} \gamma_{12}(1-d)-\mu\left(\mu+\mu_{1}+\gamma_{12}\right)\left(\mu+\mu_{22}+\gamma_{22}\right)}{\beta_{2} \gamma_{12}(1-d)\left(\mu h_{2}+1\right)\left(\mu+\mu_{1}+\gamma_{12}\right)}, \\
\tilde{I}=\frac{\beta_{2} \gamma_{12}(1-d)-\mu\left(\mu+\mu_{1}+\gamma_{12}\right)\left(\mu+\mu_{22}+\gamma_{22}\right)}{\beta_{2} \gamma_{12}(1-d)\left(\mu h_{2}+1\right)\left(\mu+\mu_{1}+\gamma_{12}\right)\left(\mu+\mu_{22}+\gamma_{22}\right)}
\end{array} .\right.
$$


Clearly, the endemic equilibrium point $\tilde{P}$ is positive if and only if $\bar{R}_{0}>1$.

Next, the stability properties of the disease-free equilibrium point $\tilde{P}_{0}$ and the endemic equilibrium point $\tilde{P}$ are considered.

Firstly, the Jacobia matrix of model (3.11) at the disease-free equilibrium point $\tilde{P}_{0}$ is as follows

$J_{\tilde{P}_{0}}=\left(\begin{array}{ccc}-\mu & 0 & -\frac{\beta_{2} A(1-d)}{\mu} \\ 0 & -A_{1} & \frac{\beta_{2} A(1-d)}{\mu} \\ 0 & \gamma_{12} & -A_{3}\end{array}\right)$.

The corresponding characteristic equation of model (3.11) at the disease-free equilibrium point $\tilde{P}_{0}$ is

$(\lambda+\mu)\left(\lambda^{2}+\left(A_{1}+A_{3}\right) \lambda+A_{1} A_{3}-A_{5}(1-d(3), 15)\right.$

Therefore, according to Routh - - Hurwitz rule, the disease-free equilibrium point is $\tilde{P}_{0}$ is locally asymptotically stable if and only if

$$
\begin{aligned}
& A_{1} A_{3}-A_{5}(1-d)>0 \\
& \Leftrightarrow \frac{A_{5}(1-d)}{A_{1} A_{3}}<1 \\
& \Leftrightarrow \bar{R}_{0}<1 \\
& \Leftrightarrow d>1-\frac{\mu\left(\mu+\mu_{1}+\gamma_{12}\right)\left(\mu+\mu_{22}+\gamma_{22}\right)}{\beta_{2} A \gamma_{12}}>0 .
\end{aligned}
$$

Secondly, the Jacobia matrix of model (3.11) at the endemic equilibrium point $\tilde{P}$ is as follows
According to Routh--Hurwitz rule, the endemic equilibrium point $\tilde{P}$ is locally asymptotically stable if and only if

$$
\left\{\begin{array}{l}
\tilde{B}_{1}>0, \tilde{B}_{3}>0, \\
\tilde{B}_{1} \tilde{B}_{2}-\tilde{B}_{3}>0 .
\end{array}\right.
$$

Clearly, the terms $\tilde{B}_{1}>0$ and $\tilde{B}_{3}>0$ hold if $\tilde{R}_{0}>1$ or $d<1-\frac{\mu\left(\mu+\mu_{1}+\gamma_{12}\right)\left(\mu+\mu_{22}+\gamma_{22}\right)}{\beta_{2} A \gamma_{12}}$.

Again, we have

$$
\begin{aligned}
& \tilde{B}_{1} \tilde{B}_{2}-\tilde{B}_{3}>0 \\
& \Leftrightarrow \frac{\beta_{2} \gamma_{12} A(1-d)\left(\mu h_{2}+1\right)\left(A_{1}+A_{2}\right)^{2}}{\beta_{2} \gamma_{12} A h_{2}(1-d)+A_{1} A_{2}} \\
& -\frac{A_{1} A_{2} h_{2}\left(\beta_{2} \gamma_{12} A(1-d)-\mu A_{1} A_{2}\right)}{\beta_{2} \gamma_{12} A h_{2}(1-d)+A_{1} A_{2}}>0 \\
& \Leftrightarrow \frac{\left(\mu h_{2}+1\right)\left(\gamma_{12} A(1-d)\left(A_{1}^{2}+A_{2}^{2}+A_{1} A_{2}\right)+\mu A_{1}^{2} A_{2}^{2}\right)}{\beta_{2} \gamma_{12} A h_{2}(1-d)+A_{1} A_{2}}>0 .
\end{aligned}
$$

Based on the above analysis, we obtain the following results.

Proposition 3.4.1 Suppose that $\beta_{2} A \gamma_{12}>\mu(\mu+$ $\left.\mu_{1}+\gamma_{12}\right)\left(\mu+\mu_{22}+\gamma_{22}\right)$, we have

(1). If $\bar{R}_{0}>1$ or $d<1-\frac{\mu\left(\mu+\mu_{1}+\gamma_{12}\right)\left(\mu+\mu_{22}+\gamma_{22}\right)}{\beta_{2} A \gamma_{12}}$, then the endemic equilibrium point $\tilde{P}$ is locally asymptotically stable,

$J_{\tilde{P}}=\left(\begin{array}{ccc}-\mu-\frac{\beta_{2} A(1-d) \tilde{I}}{1+\beta_{2}(1-d) h_{2} \tilde{I}} & 0 & \frac{\beta_{2}(1-d)\left(\left(\mu+\mu_{1}+\gamma_{12}\right)\left(\mu+\mu_{22}+\gamma_{22}\right)-\gamma_{12} A\right)}{\mu \gamma_{12}\left(1+\beta_{2}(1-d) h_{2} \tilde{I}\right)^{2}} \\ \frac{\beta_{2} A(1-d) \tilde{I}}{1+\beta_{2}(1-d) h_{2} \tilde{I}} & -\left(\mu+\mu_{1}+\gamma_{12}\right) & \frac{\beta_{2}(1-d)\left(\gamma_{12} A-\left(\mu+\mu_{1}+\gamma_{12}\right)\left(\mu+\mu_{22}+\gamma_{22}\right)\right)}{\mu \gamma_{12}\left(1+\beta_{2}(1-d) h_{2} \tilde{I}\right)^{2}} \\ 0 & \gamma_{12} & -\left(\mu+\mu_{22}+\gamma_{22}\right)\end{array}\right)$.

The corresponding characteristic equation of model (3.11) at the endemic equilibrium point $\tilde{P}$ is

$$
\lambda^{3}+\tilde{B}_{1} \lambda^{2}+\tilde{B}_{2} \lambda+\tilde{B}_{3},
$$

where

$$
\left\{\begin{array}{l}
\tilde{B}_{1}=\frac{\beta_{2} \gamma_{12} A(1-d)\left(\mu h_{2}+1\right)}{\beta_{2} \gamma_{12} h_{2}(1-d)+A_{1} A_{2}}+A_{1}+A_{2} \\
\tilde{B}_{2}=\frac{A_{1} A_{2} h_{2}\left(\beta_{2} \gamma_{12} A(1-d)-\mu A_{1} A_{2}\right)+\beta_{2} \gamma_{12} A(1-d)\left(\mu h_{2}+1\right)\left(A_{1}+A_{2}\right)}{\beta_{2} \gamma_{12} h_{2}(1-d)+A_{1} A_{2}} \\
\tilde{B}_{3}=\frac{A_{1} A_{2}\left(\mu h_{2}+1\right)\left(\beta_{2} \gamma_{12} A(1-d)-\mu A_{1} A_{2}\right)}{\beta_{2} \gamma_{12} h_{2}(1-d)+A_{1} A_{2}} .
\end{array}\right.
$$


Proposition 3.4.1 reveals that COVID-19, which incorporates the relatively high infection rate, can be controlled while the contacting distance between the healthy individuals and the symptomatic infected individuals is larger than the threshold value $\bar{d}^{*}=$ $1-\frac{\mu\left(\mu+\mu_{1}+\gamma_{12}\right)\left(\mu+\mu_{22}+\gamma_{22}\right)}{\beta_{2} A \gamma_{12}}$. This threshold value decreases with the decrease in the immigration rate of susceptible population. That is to say, the contacting distance could be shortened in the locations with the low population density. The equivalent results of Proposition 3.4.1, which take the immigration rate of susceptible population as the controlling parameter, can also be proposed as follows.

Proposition 3.4.2 We have the following conclusions

(1) If $0<A<\frac{\mu\left(\mu+\mu_{1}+\gamma_{12}\right)\left(\mu+\mu_{22}+\gamma_{22}\right)}{\beta_{2} \gamma_{12}(1-d)}$, then the disease-free equilibrium point $\tilde{P}_{0}$ is locally asymptotically stable,

(2). If $\frac{\mu\left(\mu+\mu_{1}+\gamma_{12}\right)\left(\mu+\mu_{22}+\gamma_{22}\right)}{\beta_{2} \gamma_{12}(1-d)}<A<1$, then the endemic equilibrium point $\tilde{P}$ is locally asymptotically stable.

Proposition 3.4.2 reveals that the COVID-19 can be controlled while the immigration rate of susceptible population is smaller than the threshold value $\bar{A}^{*}=$ $\frac{\mu\left(\mu+\mu_{1}+\gamma_{12}\right)\left(\mu+\mu_{22}+\gamma_{22}\right)}{\beta_{2} \gamma_{12}(1-d)}$, and this threshold value increases with the increase in the contacting distance between the healthy individuals and the symptomatic infected individuals. That is to say, only if the contacting distance is large enough, the relatively large immigration of local peoples cannot induce the second outbreak of the COVID-19.

\section{A case study}

Since from January 20, 2020, all data (including information on new cases, cumulative cases, suspected cases, recovered cases and death cases) were provided by National Health Commission of the People's Republic of China (NHPRC). As for Wuhan city in which the first case was reported, the corresponding data were restricted by many factors, such as the awareness of the epidemiological characteristics of the new coronavirus, the severe lack of medical resources, the processing and fusion capabilities of reported data and

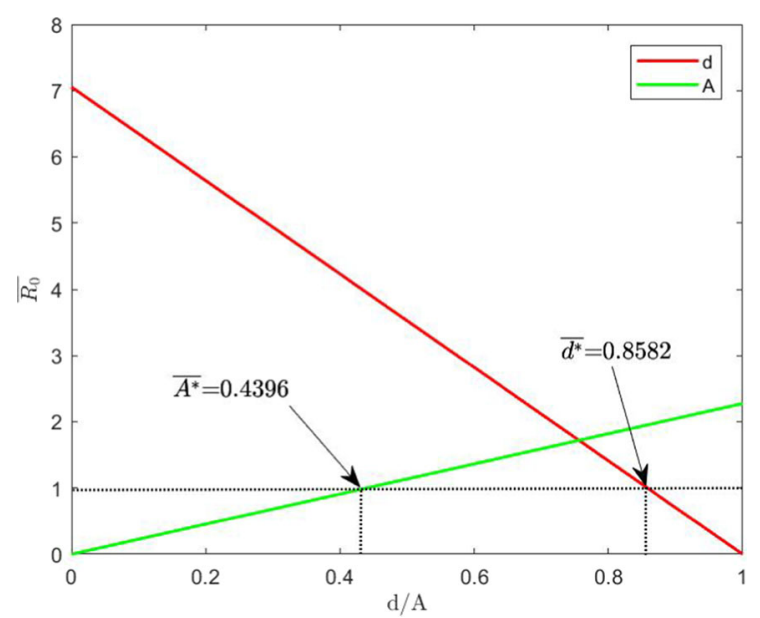

Fig. 1 The change tendency of $\bar{R}_{0}$ with $d$ and $A$

insufficient nucleic acid detection capabilities. However, the Wuhan Municipal Government revised some of the final statistical data April 17, 2020 the daily case data have not been revised.

Noting the specific characteristics of the reported data in Wuhan city, data of daily new cases from January 1, 2020, to March 18, 2020, were reported based on the data confirmed by laboratory calculation and testing in the government's statutory epidemic information reporting system $[2,10,12]$. The results in Hao et al. [22] showed that the diagnosis rate of COVID-19 in Wuhan during this period was 0.13 . In this paper, based on the statistic factors such as the calculation and detection capabilities, and the authenticity of data before January 1, 2020, the data from January 1, 2020 to March 18, 2020, are adopted.

By the method of Approximate Bayesian Computation Sequential Monte Carlo (ABC-SMC) to fit model (3.11), all parameters are estimated in Table 1. According to the formula (3.13) and the technically selected parameter values, the threshold value of the contacting distance $\bar{d}^{*}$ is 0.8582 and the threshold value of the influx of the susceptible subpopulation $\bar{A}^{*}$ is 0.4396 . The basic reproduction number of COVID19 decreases with increase in the contacting distance between the susceptible individuals, and the asymptomatic infected individuals increases and decreases in the influx of the susceptible subpopulation. The specific distribution is shown in Fig. 1. Figure 1 shows that the basic reproduction number $\bar{R}_{0}$ is less than one if the contacting distance is larger than its threshold 0.8582 


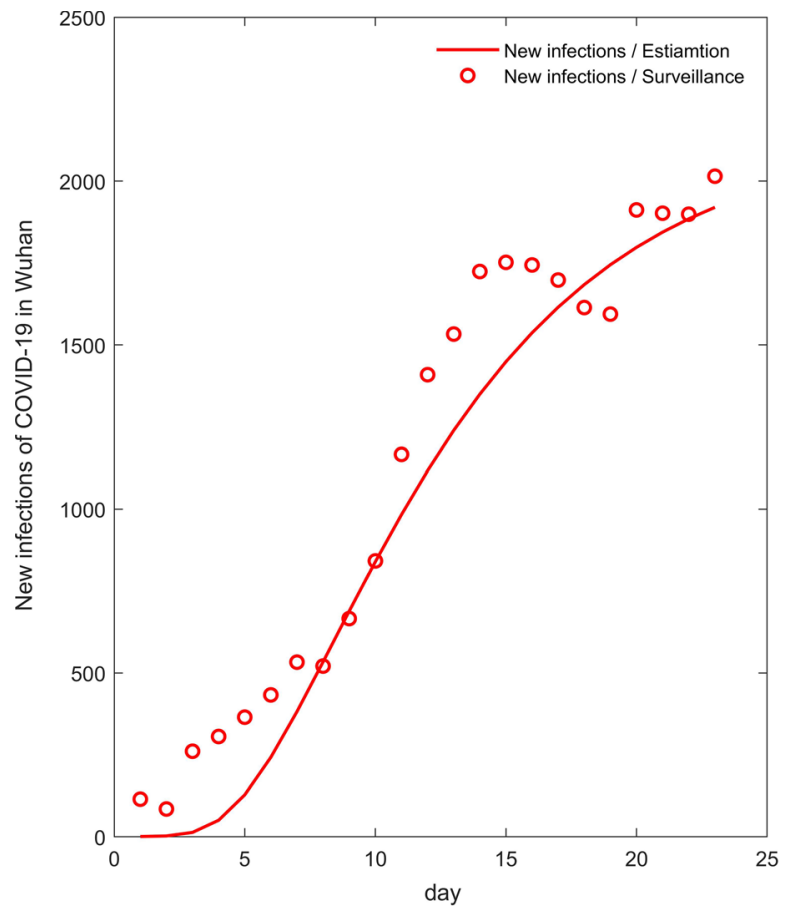

Fig. 2 Estimation and surveillance of the new infected cases

and/or the threshold value of the influx of the susceptible subpopulation is less than its threshold 0.4396 . Therefore, COVID-19 will be theoretically controlled if the contacting distance is larger than $0.8582 \mathrm{and} / \mathrm{or}$ the influx of the susceptible subpopulation is less than 0.4396 .

For Wuhan city, before January 23, 2020, the basic reproduction number is 3.52 which is larger than one, this means that COVID-19 will be endemic disease if any control measures are not implemented. However, after January 23, 2020, the initial government interventions including keeping the contacting distance and prohibiting into/out Wuhan City are adopted, then the basic reproduction number is 0.31 which is less than one. Two periods of COVID-19 in Wuhan city are evidently revealed and the specific distribution is clearly shown in Fig. 2. The initial government intervention period from January 8 to January 22, 2020, is shown in the left in Fig. 2. The lockdown period and the pharmaceutical interventions stage from January 23 to March 18, 2020, is shown in the right in Fig. 2. Therefore, the effective measures, such as decrease in the influx of the susceptible subpopulation and increase in the contacting distance between the susceptible individuals and

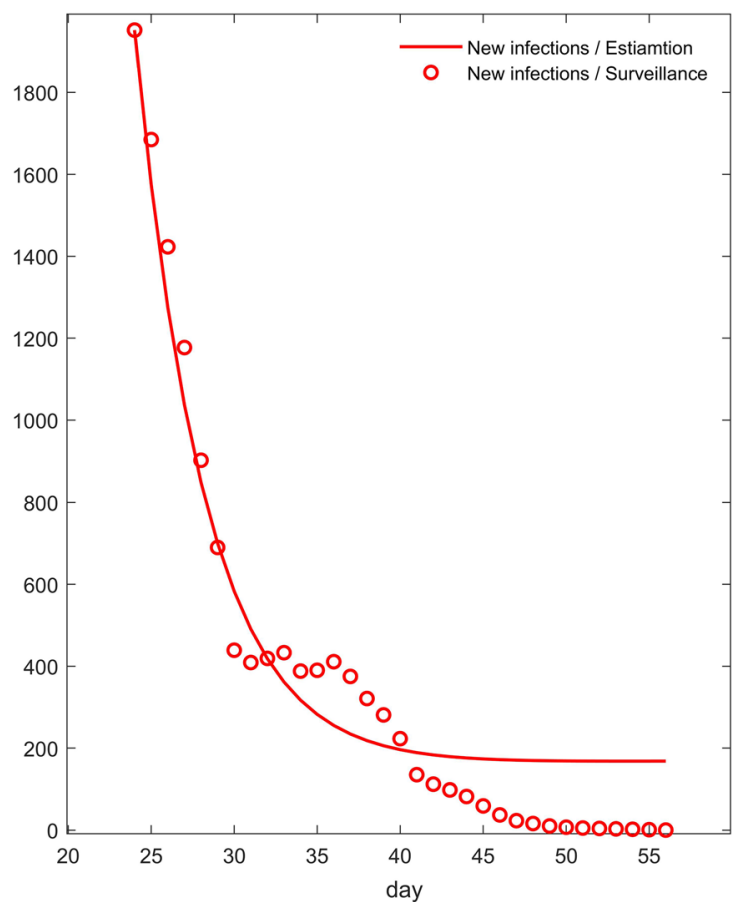

the asymptomatic infected individuals, are important to control COVID-19.

The following sensitivity analysis and numerical simulation show that these two control measures are the effective measures for preventing the transmission of COVID-19.

\subsection{Sensitivity analysis}

In this section, the sensitivity analysis for model (3.11) is conducted based on the reported cases of the COVID19 in Wuhan city and the estimated parameters in Table 1.

In order to identify the key factors which affect the change of the infectious cases, this paper applies Latin Hypercube Sampling (LHS) for parameter sampling and Paranoid Rank Correlation Coefficient (PRCC) method for sensitivity analysis of the parameters in model (3.11). When performing parameter sampling, we choose uniform distribution as the prior distribution, sampling times $N=1000$, the reference values of the parameters of model (3.11) are given in Table 1, and the value range is $20 \%$ up and down the reference 


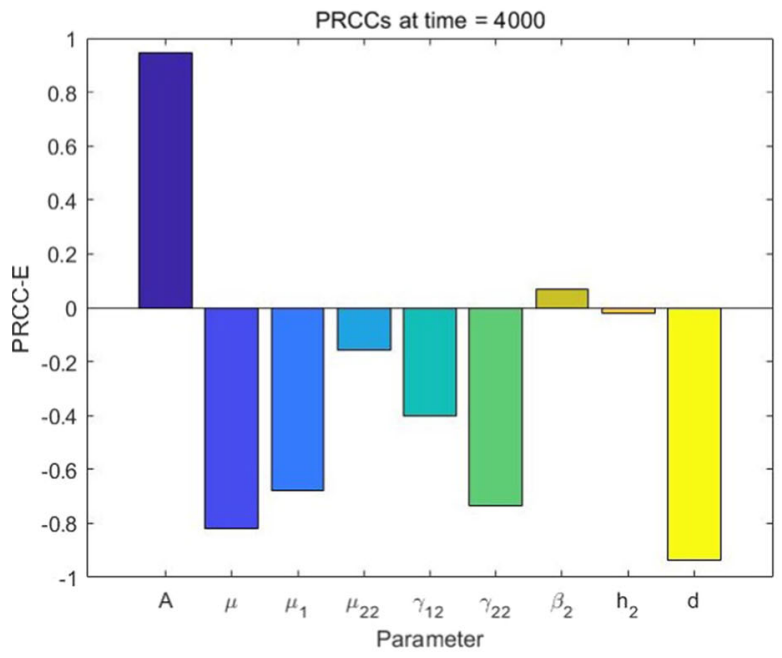

(a)

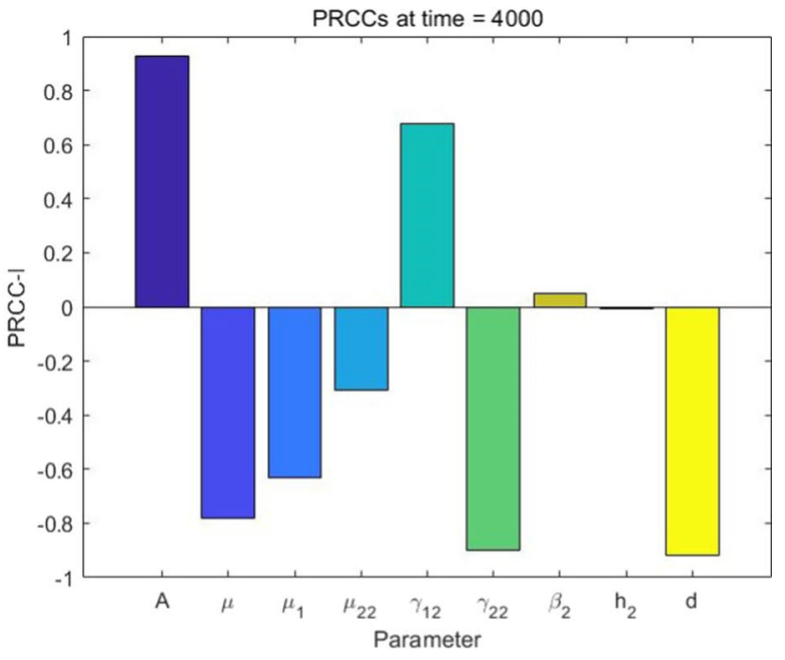

(b)

Fig. 3 PRCC values of parameters in $E(t)$ and $I(t)$ at $t=4000$
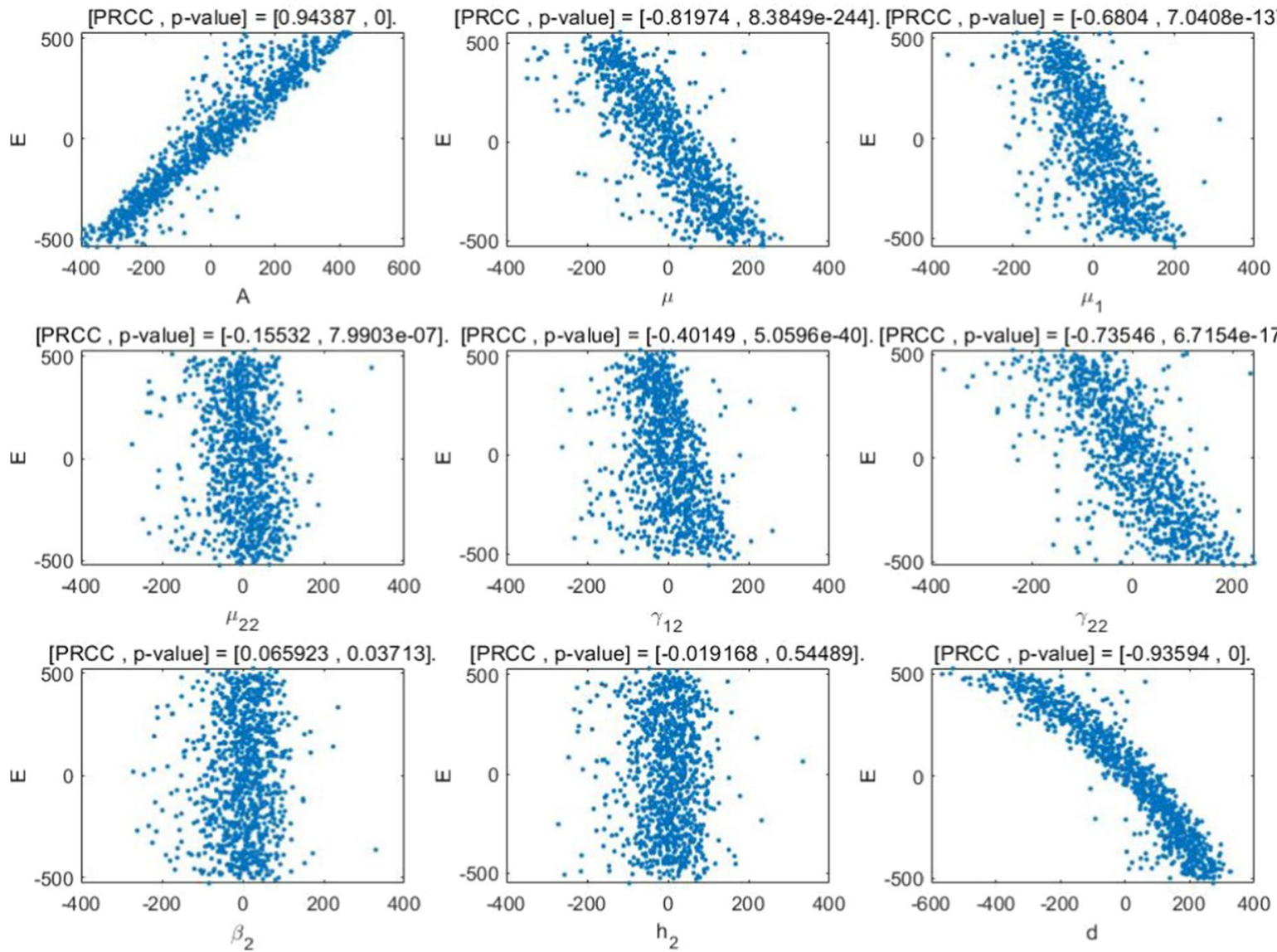

Fig. 4 Scatter plot of PRCC value for each parameter in $E(t)$ 
Table 2 The estimated values of parameters in model (3.11)

\begin{tabular}{|c|c|c|c|}
\hline Parameter & Definitions & Estimated mean value & Data source \\
\hline A & $\begin{array}{l}\text { The influx of the susceptible } \\
\text { subpopulation }\end{array}$ & Variable & $A \geq 0$ \\
\hline$\beta_{2}$ & The intrinsically infectious rate & 0.8080 & ABC-SMC \\
\hline$h_{2}$ & $\begin{array}{l}\text { The average outdoor time of each } \\
\text { symptomatic infected individual }\end{array}$ & 0.5062 & $\mathrm{ABC}-\mathrm{SMC}$ \\
\hline$\mu$ & $\begin{array}{l}\text { The natural death rates of all } \\
\text { subpopulations }\end{array}$ & 0.0146 & WHO \\
\hline$\mu_{1}$ & $\begin{array}{l}\text { The recovery rate of the incubation } \\
\text { subpopulation }\end{array}$ & 0.1398 & ABC-SMC \\
\hline$\mu_{22}$ & $\begin{array}{l}\text { The death rate induced by the coronavirus } \\
\text { disease } 2019 \text { (COVID-19) }\end{array}$ & 0.0402 & ABC-SMC \\
\hline$\gamma_{12}$ & $\begin{array}{l}\text { The transition rate from the incubation } \\
\text { subpopulation to the symptomatic } \\
\text { infected subpopulation }\end{array}$ & 0.1513 & ABC-SMC \\
\hline$\gamma_{22}$ & $\begin{array}{l}\text { The recovery rate of the symptomatic } \\
\text { infected subpopulation }\end{array}$ & 0.3302 & ABC-SMC \\
\hline$d$ & $\begin{array}{l}\text { The contacting distance between the } \\
\text { susceptible individuals and the } \\
\text { asymptomatic infected individuals }\end{array}$ & Variable & $0 \leq d \leq 1$ \\
\hline
\end{tabular}

ABC-SMC Approximate Bayesian Computation Sequential-Monte Carlo, WHO World Health Organization

value. The parameters with $p$ value greater than 0.01 are considered to have no effect on the model.

Figure $3 \mathrm{a}$ and Table 4 show that the parameters $A$ and $E(t)$ are positively correlated, that is, the population of $E(t)$ subgroups will increase as parameter $A$ increases. The parameters $\mu, \mu_{1}, \mu_{22}, \gamma_{12}, \gamma_{22}$ and $d$ are negatively correlated with $E(t)$, that is, increasing $\mu, \mu_{1}, \mu_{22}, \gamma_{12}$, $\gamma_{22}$ and $d$ will cause $E(t)$ to decrease. Figure 3 shows that the monotonicity of the parameters $A, \mu, \mu_{1}, \gamma_{22}$ and $d$ is the most significant and $E(t)$ is mainly affected by these important parameters. Figure $3 \mathrm{~b}$ and Table 4 show that that the parameters $A$ and $\gamma_{12}$ are positively correlated with $I(t)$, that is, $I(t)$ will increase as $A$ and $\gamma_{12}$ increase. The parameters $\mu, \mu_{1}, \mu_{22}, \gamma_{22}, d$ and $I(t)$ are negatively correlated, that is, the population of $I(t)$ will decrease as the parameters $\mu, \mu_{1}, \mu_{22}$, $\gamma_{22}$ and $d$ increase. Furthermore, Fig. 4 reveals that the monotonicity of the parameters $A, \mu, \mu_{1}, \mu_{22}, \gamma_{12}, \gamma_{22}$ and $d$ is the most significant and $I(t)$ is mainly affected by these parameters.

Figure 5 reveals that the PRCC value of each parameter changes significantly in the early stage of the outbreak. Meanwhile, Fig. $5 \mathrm{~b}$ shows that the parameters $A, \mu, \mu_{1}, \gamma_{12}, \gamma_{22}$ and $d$ have undergone significant changes being related to $I(t)$. The PRCC values of the parameters $A$ and $d$ show a trend of first increasing, then decreasing and finally increasing again. This is induced by the rapid increase in the number of patients after the outbreak, and the susceptible subpopulation $S(t)$ is more likely to be infected under the small contacting distance with the initial outbreak. At this time, the contacting distance will have a greater impact on the number of patients. With the improvement of people's awareness of the prevention of epidemics and medical conditions, people's behavior of reducing the outdoor time and using masks will lead to less and less impact of $d$ on $I(t)$. According to the phased results of the epidemic prevention work, people's awareness of epidemic prevention will become weaker than before. At this time, the influence of the parameters $A$ and $d$ on $I(t)$ will increase.

Figure 6 and Table 5 show the influence of each parameter on the basic reproduction number $R_{0}$. The parameters $A, \beta_{2}$ and $R_{0}$ are positively correlated, and the parameter $A$ has a greater impact on $R_{0}$. $R_{0}$ will increase rapidly as the parameter $A$ increases, and more susceptible people will be infected. The parameters $\mu$, $\mu_{1}, \gamma_{22}$ and $d$ are negatively correlated with $R_{0}$. Most importantly, $R_{0}$ will decrease rapidly as the parameter $d$ increases, and the maximum value of PRCCs is 

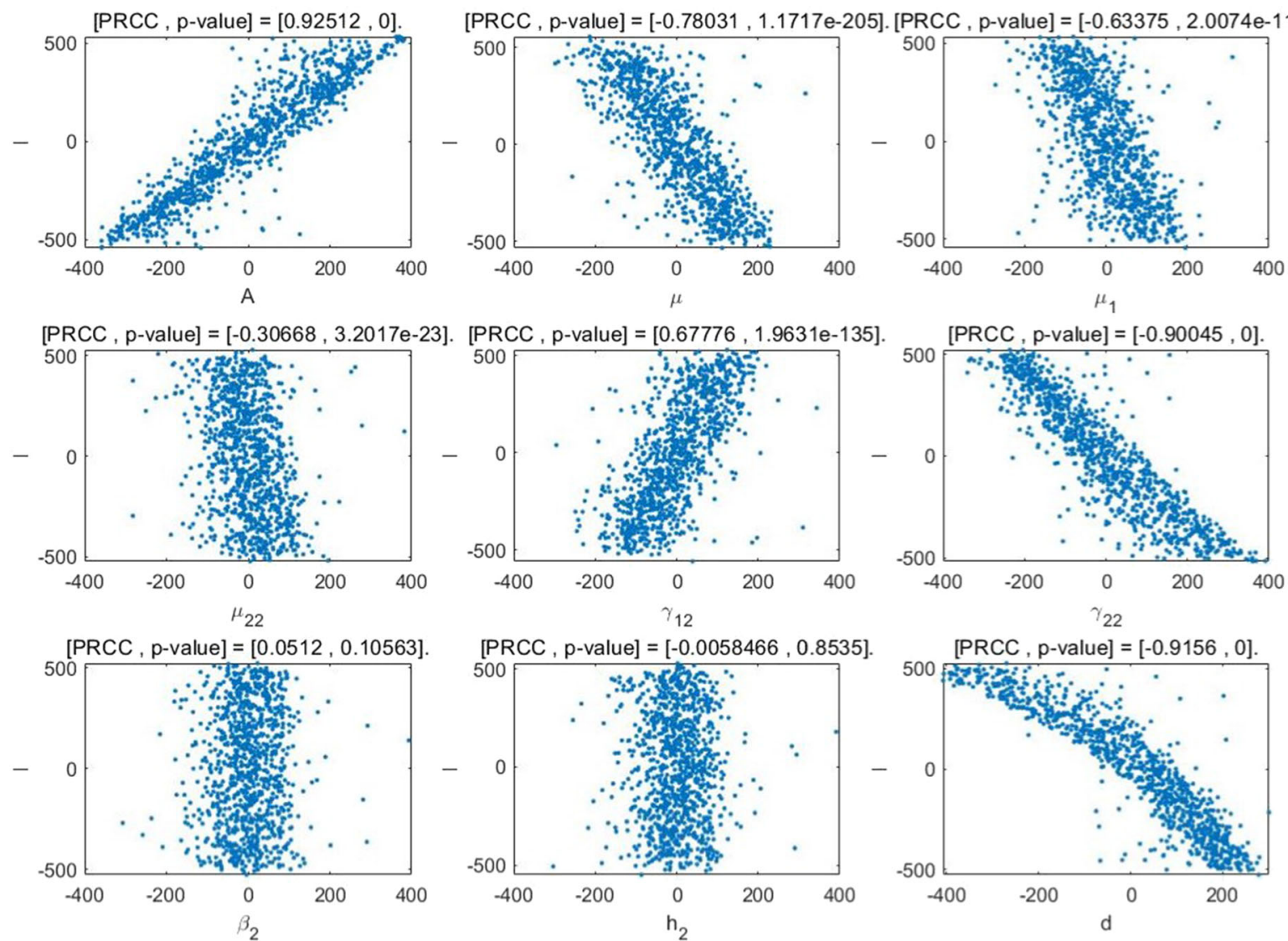

Fig. 5 Scatter plot of PRCC value for each parameter in $I(t)$

Table 3 PRCC value and $P$ value of each parameter in $E(t)$

\begin{tabular}{llllll}
\hline Parameter & PRCC values & $P$ value & Parameter & PRCC values & $P$ value \\
\hline$A$ & 0.9438 & 0.0000 & $\gamma_{22}$ & -0.7352 & 0.0000 \\
$\mu$ & -0.8197 & 0.0000 & $\beta_{2}$ & -0.0660 & 0.0376 \\
$\mu_{1}$ & -0.6803 & 0.0000 & $h_{2}$ & -0.0191 & 0.5471 \\
$\mu_{22}$ & -0.1551 & 0.0000 & $d$ & -0.09359 & 0.0000 \\
$\gamma_{12}$ & -0.4012 & 0.0000 & & & \\
\hline
\end{tabular}

Table 4 PRCC value and $P$ value of each parameter in $I(t)$

\begin{tabular}{lccccc}
\hline Parameter & PRCC values & $P$ value & Parameter & PRCC values & $P$ value \\
\hline$A$ & 0.9251 & 0.0000 & $\gamma_{22}$ & -0.9004 & 0.0000 \\
$\mu$ & -0.7803 & 0.0000 & $\beta_{2}$ & 0.0512 & 0.1071 \\
$\mu_{1}$ & -0.6338 & 0.0000 & $h_{2}$ & -0.0058 & 0.8544 \\
$\mu_{22}$ & -0.3067 & 0.0000 & $d$ & -0.9156 & 0.0000 \\
$\gamma_{12}$ & 0.6778 & 0.0000 & & & \\
\hline
\end{tabular}


Table 5 PRCC value and $P$ value of each parameter in $R_{0}$

\begin{tabular}{llllll}
\hline Parameter & PRCC values & $P$ value & Parameter & PRCC values & $P$ value \\
\hline$A$ & 0.3502 & 0.0000 & $\gamma_{22}$ & -0.2578 & 0.0000 \\
$\mu$ & -0.3727 & 0.0000 & $\beta_{2}$ & 0.3472 & 0.0000 \\
$\mu_{1}$ & -0.0943 & 0.0000 & $h_{2}$ & -0.0066 & 0.7666 \\
$\mu_{22}$ & -0.0406 & 0.0696 & $d$ & -0.6973 & 0.0000 \\
$\gamma_{12}$ & 0.1514 & 0.0000 & & & \\
\hline
\end{tabular}

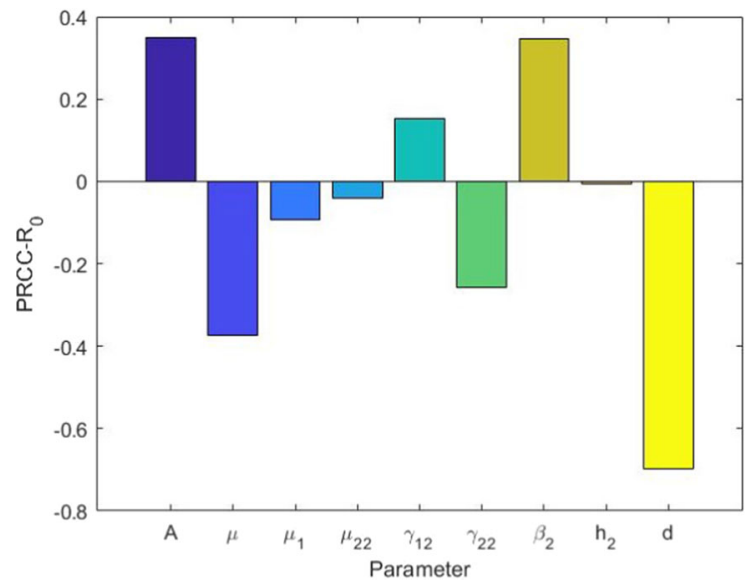

Fig. 6 PRCC values of each parameter in $R_{0}$

$|P R C C(d)|=0.6973$ which indicates that $d$ has the greatest impact on $R_{0}$. In other words, as long as $d$ is controlled within a certain range, $R_{0}$ can be clearly

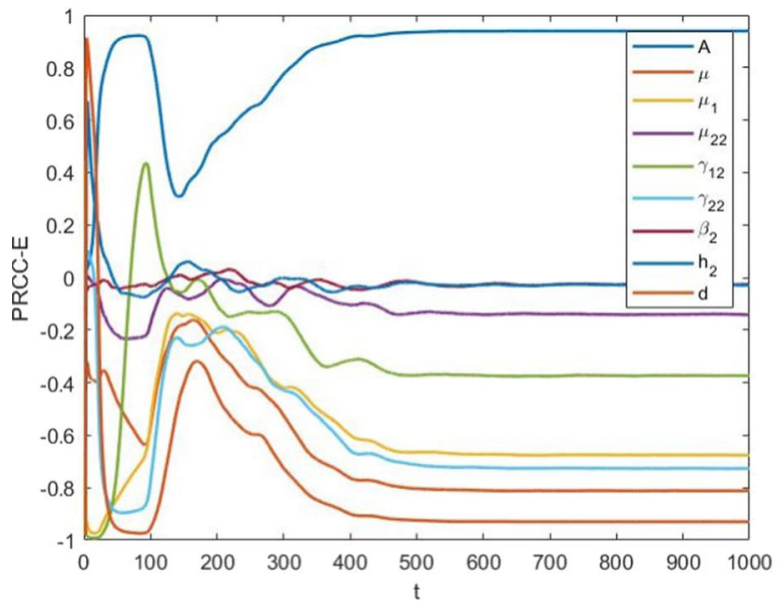

(a) minimized. The changing tendency of the PRCC value of each parameter over time is shown in Fig. 7. Figure 7 reveals that the sensitivity is convergent, and thus the robustness and stability of the sensitivity analysis are guaranteed.

\subsection{Numerical simulation}

Figures 8, 9 and 10 show that COVID-19 will be ultimately controlled when the contacting distance is larger than the threshold value 0.8582 . Figure 8 selects the contacting distance between the susceptible individuals and the symptomatic infected individuals as 0.91 which is larger than the threshold value 0.8582 , and hence COVID-19 will be vanished. Since the parameter $d$ is normalized in the modeling processes, the COVID-19 will be completely controlled only if the susceptible individuals and the symptomatic infected individuals keep their contacting distance to be larger

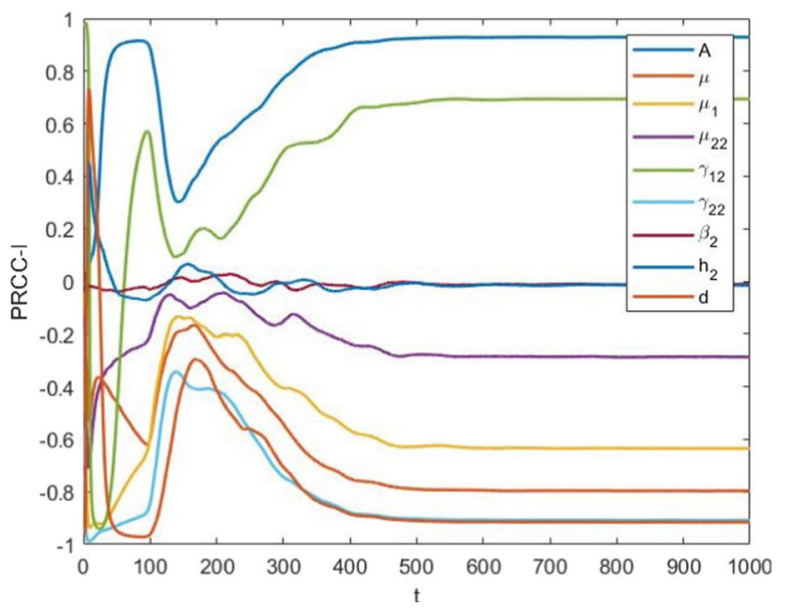

(b)

Fig. 7 PRCC value of each parameter over time 


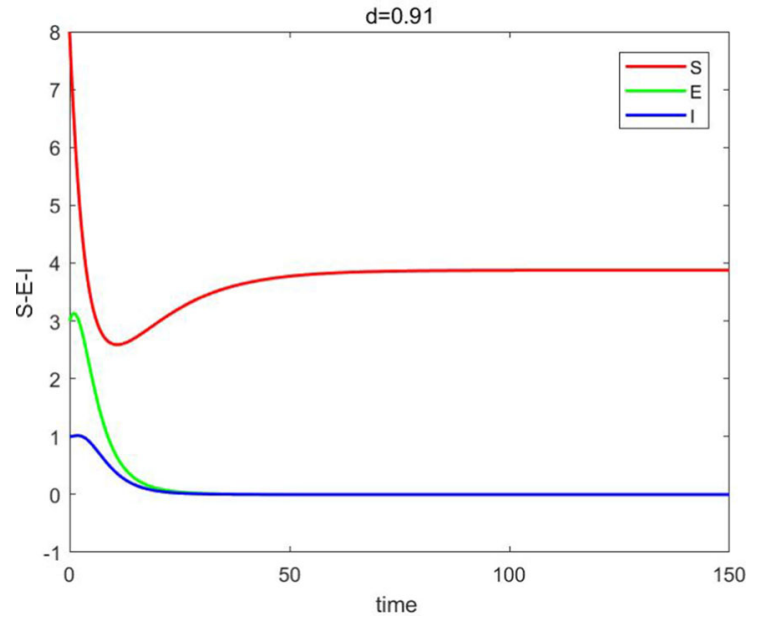

Fig. 8 The disease-free equilibrium point is asymptotically stable with $d=0.91$

than $0.8582 \times D$, where $D$ is the spreading distance of the coronavirus of COVID-19 when people are breathing and/or sneezing under certain environment. The World Health Organization (WHO) claimed that the spreading distance of the coronavirus of COVID-19 is more than 2 meters and even 4.5 meters under some certain environmental conditions. Hence, individuals should be required to keep away at least 1.7 meters rather than 1.5 meters which is adopted by many disease control departments of some countries. Figure 9 reveals that COVID-19 will be vanished as long as the contacting distance is larger than the threshold value 0.8582 , and the extinct lag of COVID-19 closely relates to the contacting distance after January $23^{t} h$, 2020. Furthermore, Fig. 9 shows that the extinct lags decrease of the incubation infected individuals and the symptomatic infected individuals as the contacting distance increase. Figure 9a and $b$ reveals that the minimum extinct lag of the incubation infected individuals is larger than that of the symptomatic infected individuals. Figure 10 reveals that COVID-19 will be vanished when the contacting distance is larger than its threshold value although the immigration rates are less than the threshold value 0.4396 , and the extinct lag of COVID-19 has the close relationship with the people's immigration to Whan city. Furthermore, the extinct lag decreases with the people's immigrating number to the streets of Wuhan city decreasing. Figure 10a and $\mathrm{b}$ shows that the minimum extinct lag of the incubation and the symptomatic infected individuals is smaller than that of the symptomatic infected individuals when the people's immigrating number to the streets from their own home to Wuhan city is $10 \%$ of the resident populations of Wuhan city after January 23, 2020. Moreover, the extinct lag of COVID-19 will become longer when the immigration rate increases. Most interestingly, if the active individuals of the residents of Wuhan city keep the safe contacting distance, their outdoor time of has no influence on the extinction of COVID-19. This implies that some individuals could move outdoors as long as they do not gather and can keep just far enough. Therefore, it is reasonable to sug-

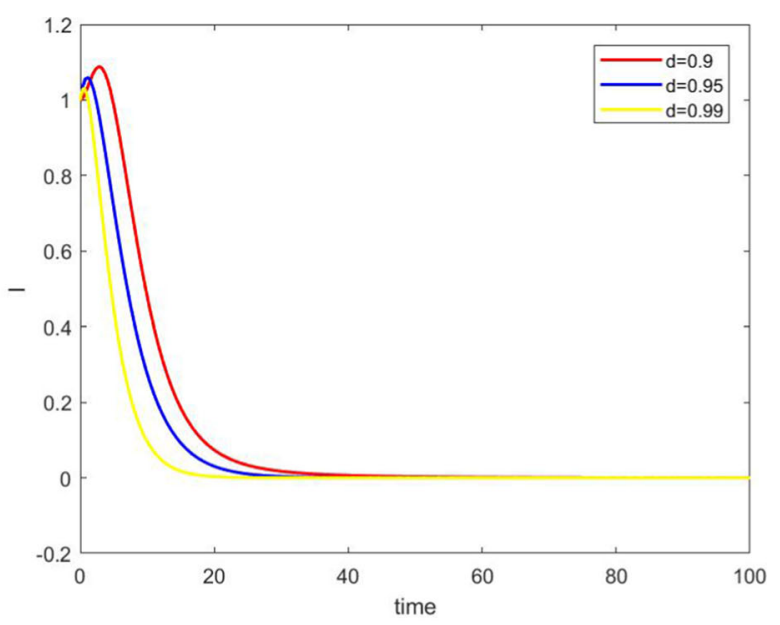

(b)

Fig. 9 The COVID-19 will be vanished while the contacting distances is larger than the threshold value 0.8582 


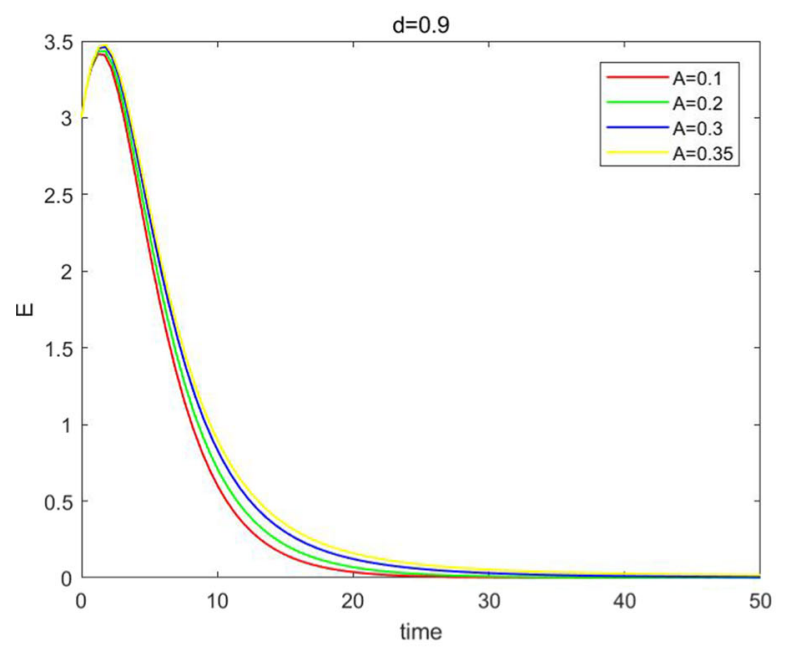

(a)

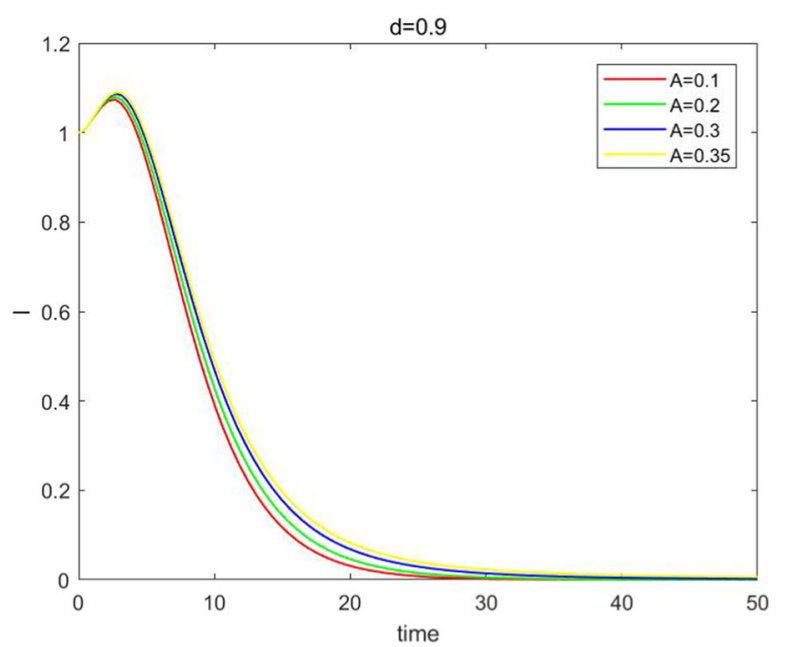

(b)

Fig. 10 The COVID-19 will be vanished with $d=0.9$ although the immigration rates are smaller than the threshold value 0.4396

gest that the necessary and safe outdoor activities of the healthy individuals could not induce COVID-19 to be endemic again. Actually, this suggestion is effectively adopted by many countries.

Figures 11, 12 and 13 show that COVID-19 may become endemic disease when the contacting distance is smaller than the threshold value 0.8582 . Figure 11 reveals that COVID-19 will become endemic when the contacting distance is smaller than the threshold value 0.8582 . Figure 12 reveals that the endemic lag decreases as the contacting distance decreases.

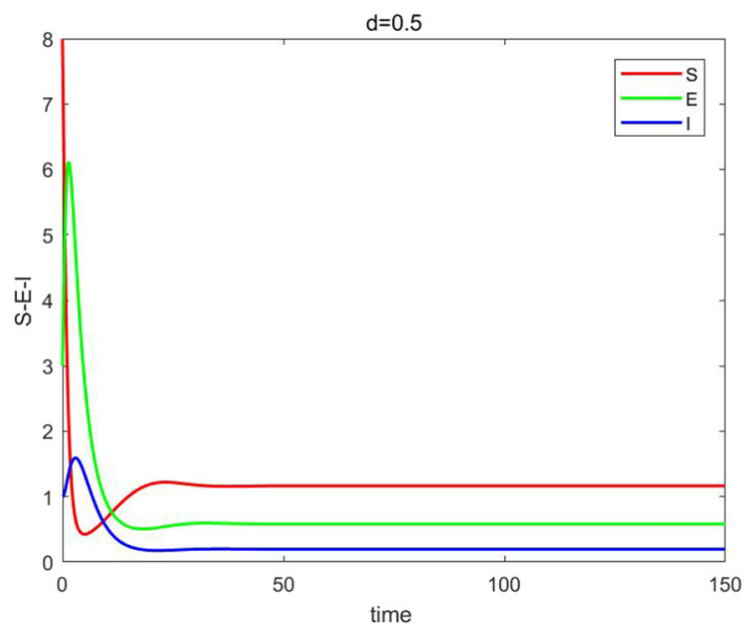

Fig. 11 The endemic equilibrium point is asymptotically stable with $d=0.5$
Hence, the contacting distance should be increased in order to delay the outbreak of COVID-19. Moreover, Fig. 12a and $\mathrm{b}$ shows the minimum endemic lag of the symptomatic infected individuals larger than that of the incubation and symptomatic infected individuals. This means that the symptomatic infected individuals should be exactly and completely treated by the non-pharmaceutical interventions or the pharmaceutical interventions. Figure 13a and b shows that, although the immigrating individuals to the streets from their own home in Wuhan city is smaller than $43.96 \%$ of the residents of Wuhan city, COVID-19 may become endemic disease after 28 like-days (two incubation lags) while the contacting distance is 40 percent of the spreading distance of COVID-19.

\section{Discussion and conclusion}

In this paper, an SEIR-type epidemic model for COVID-19 incorporating the contacting distance between the susceptible and asymptomatic/symptomatic infected individuals and the influx of the susceptible subpopulation is presented. The contacting distance and the influx of the susceptible subpopulation are explicitly incorporated into the incidence function to consider its influences on the spread of COVID19. Our results show that the contacting distance and the influx of the susceptible subpopulation play an important role in controlling COVID-19. Furthermore, 


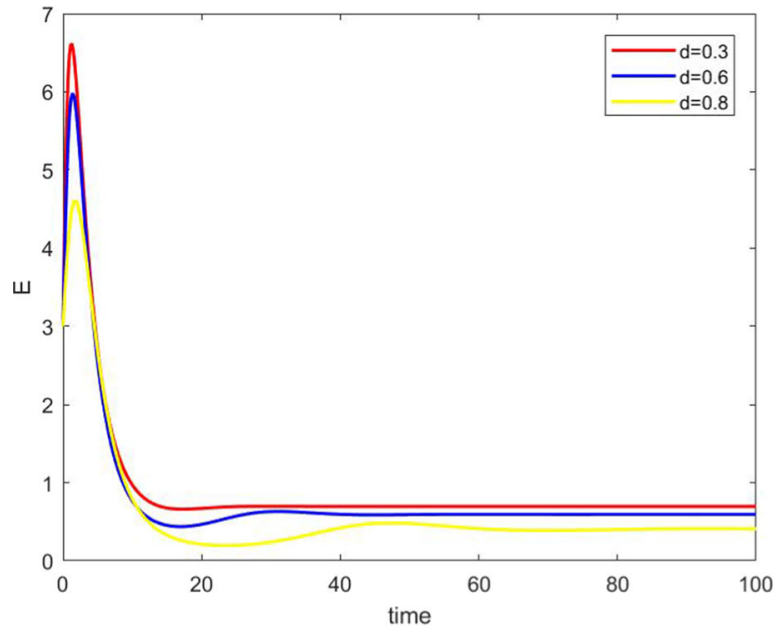

(a)

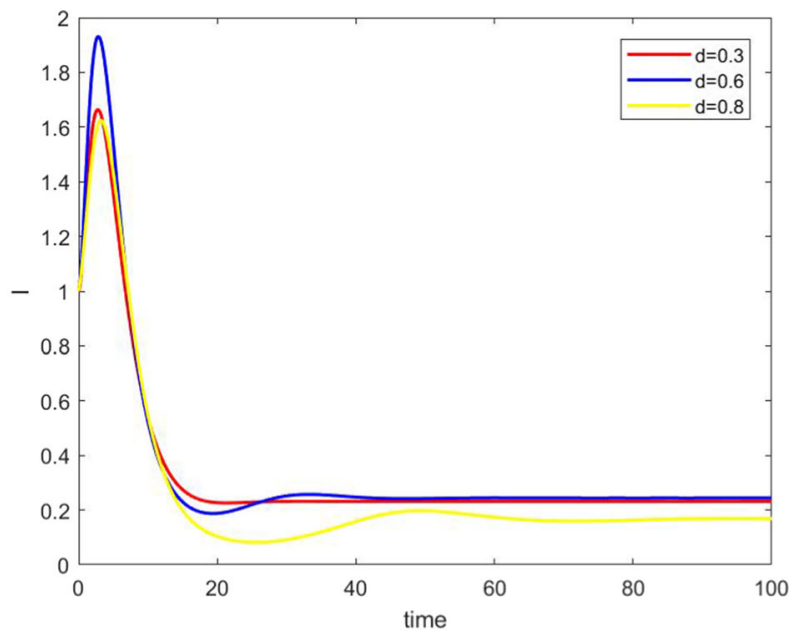

(b)

Fig. 12 The COVID-19 will be endemic while the contacting distances are smaller than the threshold value 0.8582

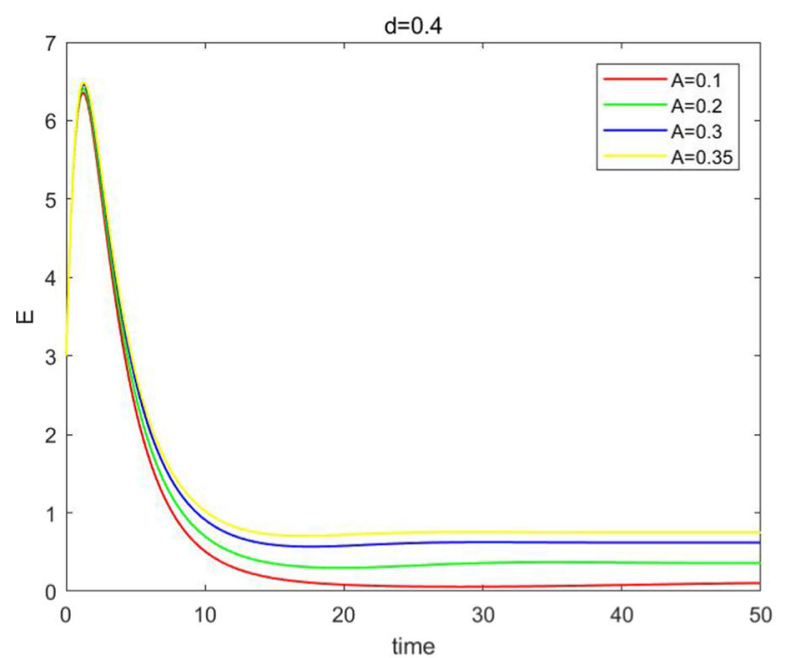

(a)

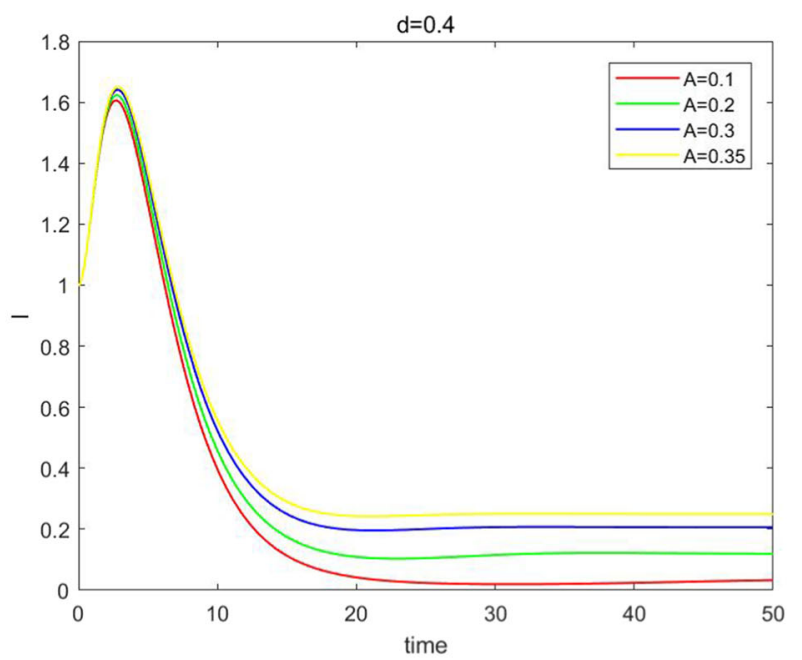

(b)

Fig. 13 The COVID-19 will be endemic with $d=0.4$ although the immigration rates are smaller than the threshold value 0.4396

the corresponding threshold values of the contacting distance and the influx of the susceptible subpopulation, which could induce COVID-19 to be extinct or endemic, are obtained by mathematical analysis based on the ordinary differential equation. Meanwhile, all parameters of the presented model are estimated based on the data of Wuhan city, and the threshold values of the contacting distance and the immigration rate are obtained. The theoretical and numerical conclusions show that COVID-19 will be completely controlled when the contacting distance is larger than the corresponding threshold value $\bar{d}^{*}=1-$ $\frac{\mu\left(\mu+\mu_{1}+\gamma_{12}\right)\left(\mu+\mu_{22}+\gamma_{22}\right)}{\beta_{2} A \gamma_{12}}=0.8582$ and/or the immigration rate is smaller than the corresponding threshold value $\bar{A}^{*}=\frac{\mu\left(\mu+\mu_{1}+\gamma_{12}\right)\left(\mu+\mu_{22}+\gamma_{22}\right)}{\beta_{2}(1-d)}=0.4396$. Moreover, the sensitivity analysis reveals that the contacting distance and the influx of the susceptible subpopulation are most sensitive to the transmission of COVID-19.

Our study gives some non-pharmaceutical interventions to control COVID-19 and some reasonable suggestions for the policy-makers. However, our paper uses the average contacting distance in the modeling 
processes and does not consider the temporal and spatial effects in the contacting distance. Maybe the variable contacting distance with the temporal and spatial factors is more realistic and useful. Meanwhile, our model considers the respiratory transmission and the contacting transmission in the modeling processes only and does not take into account the aerosol transmission. These will be open issues and should be generally considered in the future work.

Author contributions ZM contributed to conceptualization, project administration, writing-original draft, writing-review and editing; SW and HL contributed to writing-review and editing, supervision; XL and XL contributed to formal analysis, software, writing-original draft, writing-review and editing; $\mathrm{XH}$ and $\mathrm{HW}$ contributed to data curation, software.

Funding Information This work was supported by Natural Science Foundation of Gansu Province (Nos. 20JR5RA238, 21JR7RA165, 21JR7RA535) and the Fundamental Research Funds for the Central Universities (lzujbky-2021-54).

Availability of data and material The dataset used and/or analyzed during the current study is available from the published works.

\section{Declarations}

Conflict of interest The authors declare no conflict of interest.

Ethics approval and consent to participate The study protocol was approved by School of Mathematics and Statistics of Lanzhou University, School of Mathematics and Computer Science of Northwest Minzu University and Faculty of Science of McMaster University.

\section{Appendix}

Proof of Proposition 3.1.1 According to differential and integral theory, we have

$$
\begin{gathered}
\frac{\mathrm{d}}{\mathrm{d} t}\left[S(t) \exp \left\{\int_{0}^{t} \frac{\beta(1-d(\tau)) E(\tau)}{1+\beta(1-d(\tau)) h E(\tau)} \mathrm{d} \tau+\mu t\right\}\right] \\
=A \exp \left\{\int_{0}^{t} \frac{\beta(1-d(\tau)) E(\tau)}{1+\beta(1-d(\tau)) h E(\tau)} \mathrm{d} \tau+\mu t\right\} .
\end{gathered}
$$

That is,

$$
\begin{aligned}
& S(t) \exp \left\{\int_{0}^{t} \frac{\beta(1-d(\tau)) E(\tau)}{1+\beta(1-d(\tau)) h E(\tau)} \mathrm{d} \tau+\mu t\right\}-S(0) \\
& =\int_{0}^{t}\left[A \exp \left\{\int_{0}^{t} \frac{\beta(1-d(\tau)) E(\tau)}{1+\beta(1-d(\tau)) h E(\tau)} \mathrm{d} \tau+\mu t\right\}\right] \mathrm{d} t .
\end{aligned}
$$

Hence,

$$
\begin{aligned}
S(t)= & \left\{S(0)+\int_{0}^{t}\left[A \operatorname { e x p } \left\{\int_{0}^{t} \frac{\beta(1-d(\tau)) E(\tau)}{1+\beta(1-d(\tau)) h E(\tau)} \mathrm{d} \tau\right.\right.\right. \\
& +\mu t\}] \mathrm{d} t\} \\
& \times \exp \left\{-\int_{0}^{t} \frac{\beta(1-d(\tau)) E(\tau)}{1+\beta(1-d(\tau)) h E(\tau)} \mathrm{d} \tau+\mu t\right\}>0 .
\end{aligned}
$$

Similarly, the positivity of the other solutions $E(t)$, $I_{A}(t), I(t)$ and $R(t)$ can be shown.

Thus, the solution $\left(S(t), E(t), I_{A}(t), I(t), R(t)\right)$ of model (2.5) with the initial conditions (2.6) are positive on the finite region for all $t>0$.

Therefore, the positivity of all solutions of model (2.7) with the initial conditions (2.8) is also guaranteed since model (2.7) is a special case of model (2.5).

Proof of Proposition 3.1.2 Define $N(t)=S(t)+$ $E(t)+I_{A}(t)+I(t)+R(t)$ and add all equations of model (2.5), it is obtained that

$$
\frac{\mathrm{d} N}{\mathrm{~d} t}=A-\mu N-\mu_{1} E--\mu_{21} I_{A}-\mu_{22} I \leq A-\mu N .
$$

By comparison theorem, it follows that

$0 \leq N(t) \leq \frac{A}{\mu}+N(0) e^{-d t}$.

Thus,

$\limsup _{t \rightarrow+\infty} N(t) \leq \frac{A}{\mu}$

Hence, all solutions $\left(S(t), E(t), I_{A}(t), I(t), R(t)\right)$ of model (2.3) with the initial conditions (2.4) are bounded on the finite region and the set $\Omega$ is a positively invariant set for model (2.3) with the initial conditions (2.4).

Therefore, the boundness of all solutions of model (2.5) with the initial conditions (2.6) is also guaranteed since model (2.5) is a special case of model (2.3).

Proof of Proposition 3.3.1 We will prove the globally asymptotical stability of the disease-free equilibrium point $E_{0}$. Define the following matrices:

$W=\left(\begin{array}{c}S \\ E \\ I\end{array}\right)$, 


$$
J_{E_{0}}=\left(\begin{array}{ccc}
-\mu & 0 & -\frac{\beta_{2} A(1-d)}{\mu} \\
0 & -\left(\mu+\mu_{1}+\gamma_{12}\right) & \frac{\beta_{2} A(1-d)}{\mu} \\
0 & \gamma_{12} & -\left(\mu+\mu_{22}+\gamma_{22}\right)
\end{array}\right),
$$

and

$$
J_{E_{0}}^{1}=\left(\begin{array}{c}
A-\frac{\beta_{2}(1-d) S I}{1+\beta_{2}(1-d) h_{2} I}+\frac{\beta_{2} A(1-d) E}{\mu} \\
\frac{\beta_{2}(1-d) S I}{1+\beta(1-d) h_{2}}-\frac{\beta_{2} A(1-d) E}{\mu} \\
0
\end{array}\right) .
$$

Based on the above definitions and notations, model (2.5) nearing the disease-free equilibrium point $E_{0}$ can be rewritten as:

$\frac{\mathrm{d} W}{\mathrm{~d} t}=J_{E_{0}} W+J_{E_{0}}^{1}$.

By simple computation, the vector $J_{E_{0}}^{1}$ is nonpositive in the set $\Omega=\{(S, I) \mid S>0, I \geq 0\}$.

Hence, combining Eq. (5.1) with the non-positivity of the vector $J_{E_{0}}^{1}$ in the set $\Omega=\{(S, I) \mid S>0, I \geq 0\}$ , we have

$$
\frac{\mathrm{d} W}{\mathrm{~d} t} \leq J_{E_{0}} W
$$

According to the local stability of the disease-free equilibrium point, $E_{0}$ and all characteristic roots of matrix $J_{E_{0}}$ have negative real parts.

Therefore, the disease-free equilibrium point $E_{0}$ is globally stable by the theory of the ordinary differential equation.

\section{References}

1. Chen, Y., Liu, Q., Guo, D.: Coronaviruses: Genome structure, replication, and pathogenesis. J. Med. Virol. 92, 418$423(2020)$

2. Tang, B., Wang, X., Li, Q., Bragazzi, N.L., Tang, S., Xiao, Y., Wu, J.: Estimation of the transmission risk of the 2019$\mathrm{nCoV}$ and its implication for public health interventions. J. Clin. Med. 9(2), 462-474 (2020)

3. Kahn, J.S., McIntosh, K.: History and recent advances in coronavirus discovery. Pediatr. Infect. Dis. J. 24, 223-227 (2005)

4. Hui, D.S.C., Zumla, A.: Severe acute respiratory syndrome: historical, epidemiologic, and clinical features. Infect. Dis. Clin. North Am. 33, 869-889 (2019)

5. De Wit, E., Van Doremalen, N., Falzarano, D., Munster, V.J.: SARS and MERS: recent insights into emerging coronaviruses. Nat. Rev. Microbiol. 14, 523-534 (2016)
6. Willman, M., Kobasa, D., Kindrachuk, J.: A comparative analysis of factors influencing two outbreaks of middle eastern respiratory syndrome (MERS) in Saudi Arabia and South Korea. Viruses 11, E1119 (2019)

7. Killerby, M.E., Biggs, H.M., Midgley, C.M., Gerber, S.I., Watson, J.T.: Middle East respiratory syndrome coronavirus transmission. Emerg. Infect. Dis. 26, 191-198 (2020)

8. Kwok, K.O., Tang, A., Wei, V.W.I., Park, W.H., Yeoh, E.K., Riley, S.: Epidemic models of contact tracing: systematic review of transmission studies of severe acute respiratory syndrome and Middle East respiratory syndrome. J. Comput. Struct. Biotechnol. 17, 186-194 (2019)

9. Cheng, V.C.C., Wong, S.C., To, K.K.W., Ho, P.L., Yuen, K.Y.: Preparedness and proactive infection control measures against the emerging Wuhan coronavirus pneumonia in China. J. Hosp. Infect. 104(3), 254-255 (2020)

10. Li, Q., Guan, X., Peng, P., et al.: Early transmission dynamics in Wuhan, China, of novel Coronavirus-infected Pneumonia. N. Engl. J. Med. 382(13), 1199-1207 (2020)

11. Rothe, C., Schunk, M., Sothmann, P., et al.: Transmission of 2019-nCoV infection from an asymptomatic contact in Germany. N. Engl. J. Med. 10, 1-3 (2020)

12. Zhao, S., Lin, Q., Ran, J., et al.: Preliminary estimation of the basic reproduction number of novel coronavirus (2019$\mathrm{nCoV}$ ) in China, from 2019 to 2020: a data-driven analysis in the early phase of the outbreak. Int. J. Infect. Dis. 92, 214-217 (2020)

13. National Health Commission of the People's Republic of China (NHCPRC). Available online: http://www.nhc.gov.cn/xcs/yqtb/202001/ c5da49c4c5bf4bcfb320ec2036480627.shtml (Report on January 22th 2020)

14. National Health Commission of the People's Republic of China (NHCPRC). Available online: http://www.nhc.gov.cn/xcs/yqtb/202002/ ac1e98495cb04d36b0d0a4e1e7fab545.shtml (Report on February 112th 2020)

15. Annas, S., Pratama, M.S., Rifandi, M., Sanusi, W., Side, S.: Stability analysis and numerical simulation of SEIR model for pandemic COVID-19 spread in Indonesia, Chaos. Solitons Fractals 139, 110072 (2020)

16. Boukanjimea, B., Caraballo, T., Fatini, M.E., Khalif, M.E.: Dynamics of a stochastic coronavirus (COVID-19) epidemic model with Markovian switching, Chaos. Solitons Fractals 141, 110361 (2020)

17. Hodgetts, D., Stolte, O., Radley, A., Leggatt-Cook, C., Groot, S., Chamberlain, K.: Near and far: social distancing in domiciled characterizations of homeless people. Urban Studes 48(8), 1739-1754 (2011)

18. Bogardus, E.: Measuring social distance. J. Appl. Sociol. 9, 299-308 (1925)

19. Ma, Z., Wang, S., Li, H.: A generalized infectious model induced by the contacting distance. Nonlinear Anal-Real. 54, 103113 (2020)

20. Simeone, M., Ian, B.H., Christian, J.R., et al.: A methodology for performing global uncertainty and sensitivity analysis in systems biology. J. Theor. Biol. 254, 178-196 (2018)

21. Diekmann, O., Heesterbeek, J.A.P., Metz, J.A.P.: On the definition and the computation of the basic reproduction ratio $R_{0}$ in models for infectious diseases in heterogeneous populations. J. Math. Biol. 28(4), 365-382 (1990) 
22. Hao, X., Cheng, S., Wu, D., et al.: Reconstruction of the full transmission dynamics of COVID-19 in Wuhan. Nature 584, 420-424 (2020)
Publisher's Note Springer Nature remains neutral with regard to jurisdictional claims in published maps and institutional affiliations. 